\title{
COMPETITIVENESS OF SOUTHERN METROPOLITAN AREAS: THE ROLE OF NEW ECONOMY POLICIES
}

\author{
Rebekka M. Dudensing and David L. Barkley ${ }^{+}$
}

ABSTRACT. The concept of regional competitiveness is increasingly popular among academics and policymakers as indicated by reports that rank or grade regional economies. Competitiveness in these studies generally is measured by growth rates in population, employment, and per capita income. This paper explores the relationships between New Economy development policies (innovation, entrepreneurship, and human capital development) and changes in competitiveness outcomes for Southern metropolitan areas. Our results suggest that inputs have different associations with regional competitiveness outcomes. Regional employment growth rates are positively associated with innovation and entrepreneurship while changes in per capita income are related to measures of human capital.

Keywords: Competitiveness, New Economy, Regional Economic Growth

JEL Classification Codes: $R 1, O 21$

\section{INTRODUCTION}

Competition among regions is a popular concept as indicated by the attention given to the rankings of cities and states in the academic literature and popular press. The Beacon Hill Institute's State Competitiveness Report (Tuerck et al., 2007) and the Milken Institute's Best Performing Cities (DeVol, Bedroussian, and Kim, 2007) are just two of many recent reports that rate regions based on their perceived environments for or success in competing in the New Economy. ${ }^{1}$ Community leaders and local economic development officials cite high rankings in these reports (or movement up the rankings) as indication that existing development strategies are successful and the community is "winning" its competition with other regions to develop or attract economic activity.

Bristow (2005) and Krugman (1996) attribute the popularity of interregional competition to the fact that the language of competitiveness is the language of the business community. That is, business leaders and policy makers can project their understanding of firm-level competitiveness onto the regional economy. Policy-makers from the local to supra-national levels have adopted the notion of competitiveness because it is easy to understand, supposes regions can define their fortunes, and provides metrics against which progress can be measured (Budd and Hirmis, 2004; Bristow, 2005; Greene, Tracy, and Cowling, 2007). A veritable

\footnotetext{
* The authors thank the editors and three anonymous reviewers for their helpful comments.

${ }^{+}$Dudensing is Assistant Professor in the Department of Agricultural Economics of Texas A\&M University; Barkley is Professor in the Department of Applied Economics and Statistics and Co-director of University Center for Economic Development at Clemson University.

Contact author: Rebekka M. Dudensing, Department of Agricultural Economics, Texas AgriLife Research, Texas A\&M System, 467B Blocker Building, TAMU 2124, College Station, TX 77843-2124. E-mail: rmdudensing@ag.tamu.edu

${ }^{1}$ The New Economy is generally accepted to be a knowledge-based economy, but researchers define the New Economy many ways (Atkinson and Court, 1998; Norton, 2000). It is often associated with computers and high-technology industries. However, traditional manufacturing establishments have adapted to the New Economy by using computer networks to manage supply, production, and distribution. The New Economy is believed to fuel unprecedented economic growth (Norton, 2000).
}

(C) Southern Regional Science Association 2011.

ISSN 1553-0892

SRSA, 1601 University Avenue, PO Box 6025, Morgantown, West Virginia 26506-6025, USA. 
industry has been created to measure that progress; however, competitiveness in these studies is often simply a function of the community characteristics selected for measuring (Greene, Tracy, and Cowling, 2007).

Fisher's (2005) analysis of studies measuring competitiveness finds a host of measurement issues and a general lack of scientific rigor and impartiality. In fact, Fisher questions the very notion that competitiveness is a sensible theory for economic development. Nevertheless, he contends that studies of competiveness can be improved and offers several suggestions for such improvement. Bristow (2005) also supposes that, despite questions about whether competitiveness is superior to other theories, it has become an established model in policy and business circles. Regardless of lingering questions about the concept of competitiveness, it is incumbent upon academics to both inform the regional competitiveness debate and contribute science-based polity tools to enhance performance in the global, information-driven New Economy.

Camagni (2002) adds support for a competitiveness strategy in his argument that regional economic development is based on the principal of absolute advantage rather than comparative advantage. Under the principle of absolute advantage based on competitive advantages (and agglomeration economies), Camagni refutes the premise that each region will be afforded a specialization and a role in the national and world economies. Thus, like Greene, Tracey, and Cowling (2007), Camagni suggests that regions can either get competitive or become locked in a spiral of declining economic activity.

Bristow (2005) notes that firms, not regions, actually compete but that regions help set the stage for the firms' competitiveness. Successful regions specialize in the production of goods and services for which local firms are the efficient producers. At the same time, firm productivity is affected by the quality of the regional business environment and the region's institutions, industrial structure, and economic legacy. Barney (1991) and Ma (2000) propose that competitive advantage is based on resources that are rare, valuable, inimitable, nontradable, nonsubstitutable, and both firm- and region-specific. In addition, Ma suggests that a region may possess multiple competitive advantages that are compounded to make the region the most efficient producer of a good or service, thus providing the region an absolute advantage in its production.

Programs and policies to enhance a region's potential for competitiveness in the New Economy focus on the availability and quality of human capital, investments in and commercialization of innovative activity, and the environment for entrepreneurship and small business development (Malecki, 2004). The Southern Growth Policies Board (SGPB) is a regional leader in promoting increased roles for innovation, entrepreneurship, and human capital in state and local economic development strategies. Examples of SGPB initiatives include Innovation with a Southern Accent (Doron et al., 2006), EntrepriseSouth.biz (Taylor et al., 2007), and The Mercedes and the Magnolia: Preparing the Southern Workforce for the Next Economy (Clinton and Andrews et al., 2002). Yet, metropolitan areas in the U.S. South are relatively reluctant to shift a significant share of their economic development resources from traditional industrial development activities (e.g., branch plant recruitment and infrastructure improvements) to the New Economy programs. This reluctance derives partly from past successes in recruiting large manufacturing plants and partly from a concern by policy makers that a focus on knowledge economy activities offers little short-term economic development potential for Southern metro areas with legacies of traditional manufacturing. 
The goal of this paper is to investigate relationships between the relative competitiveness of Southern metropolitan areas and the three principal policy recommendations to enhance regional development in the New Economy (innovation, entrepreneurship, and human capital). There is no single accepted measure of regional competitiveness; thus we selected three outcome measures of the competitiveness process as indicators of success in the competition for economic activity (growth rates of metropolitan population, employment, and per capita income). We assume that the "most competitive" regions will exhibit relatively rapid growth in all three measures. That is, we infer a relationship between the New Economy policies and regional competitiveness if there is an association between these policies and regional growth rates in population, employment, and per capita income.

Our findings indicate that the selected New Economy inputs are associated with metropolitan growth in population, employment, and per capita income; however, different measures of competitiveness respond to different inputs. Specifically, we find that metro employment change is positively associated with lagged levels of innovation and entrepreneurship while income change is positively related to prior levels of human capital. Thus, regions may need to be specific in defining desired outcomes and to pursue multiple development policies in order to achieve overall economic "competitiveness" in terms of an increase in both the size of the local economy and the well-being of the area's residents.

The paper is organized as follows. In the following section, regional competitiveness is defined and conceptual and econometric models of competitiveness are presented. In Section 3, measures of inputs to the regional competitiveness process are introduced, and factor analysis is used to develop a set of relatively uncorrelated measures of New Economy policy variables and regional industrial structure. In Section 4, the estimation results from a regional adjustment model are summarized. Policy implications of the findings are suggested in Section 5.

\section{COMPETITIVENESS CONCEPTS}

\subsection{Definition}

Porter (1990) often is credited with popularizing the concept of competitiveness, which he defines as the ability of firms and industries to gain and retain a share in contested markets (Bristow, 2005; Budd and Hirmis, 2004). ${ }^{2}$ Thus, Porter (1990, 2002) measures regional competitiveness, also referred to as competitive advantage, almost exclusively in terms of the productivity of firms in the region. Krugman (1990, p. 9) concurs with Porter, and he states that "...productivity isn't everything, but in the long run, it is almost everything. A country's ability to improve its standard of living over time depends almost entirely on its ability to raise output per worker." Other researchers consider competitiveness a composite measure of economic and social outcomes. Storper (1997, p. 20) includes quality of life in addition to productivity in defining competitiveness as "...the capability of a sub-national economy to attract and maintain firms with stable or rising market shares in an activity, while maintaining or increasing standards of living for those who participate in it." Gardiner (2003) and the European Commission (1999) also acknowledge the role of quality of life in determining regional competitiveness, and they propose that regional competitiveness requires the ability both to compete in international markets and to maintain high income and employment levels. In this study, we adopt the broader

${ }^{2}$ However, Atkinson (1990), Harvey (1989), and Scott and Lodge (1985) provide earlier references to competitiveness. 
definitions of competitiveness in which regional competitiveness is reflected in both more residents and jobs and higher per capita incomes.

\subsection{Conceptual and Econometric Models}

\subsubsection{Competitiveness Conceptualized}

Greene, Tracey, and Cowling (2007) find that input-output-outcome models are prevalent in conceptualizing and measuring regional competitiveness. For example, Gardiner, Martin, and Tyler (2004) and Ireland's National Competitiveness Council (2007) configure the input-outputoutcome relationship into a pyramid. The pyramid structure is appealing from a policy perspective because it implies that regions can build on their regional characteristics and competitive advantages to achieve their target outcomes. This study adopts a similar conceptualization in which New Economy competiveness inputs and policies and local industrial structure and composition both contribute to competitiveness outcomes (Figure 1). Specifically, the base of the pyramid contains the principal inputs to regional competitiveness in the New Economy as identified in previous studies of competitiveness: innovation, human capital, and entrepreneurial environment. The middle layer includes measures of industrial legacy and structure that have been found to be associated with regional economic development: industrial composition, industrial specialization, and establishment age and size. Outcomes of the competitiveness process include income, jobs, quality of life, and sustainable development.

\section{FIGURE 1. Regional Competitiveness Pyramid}

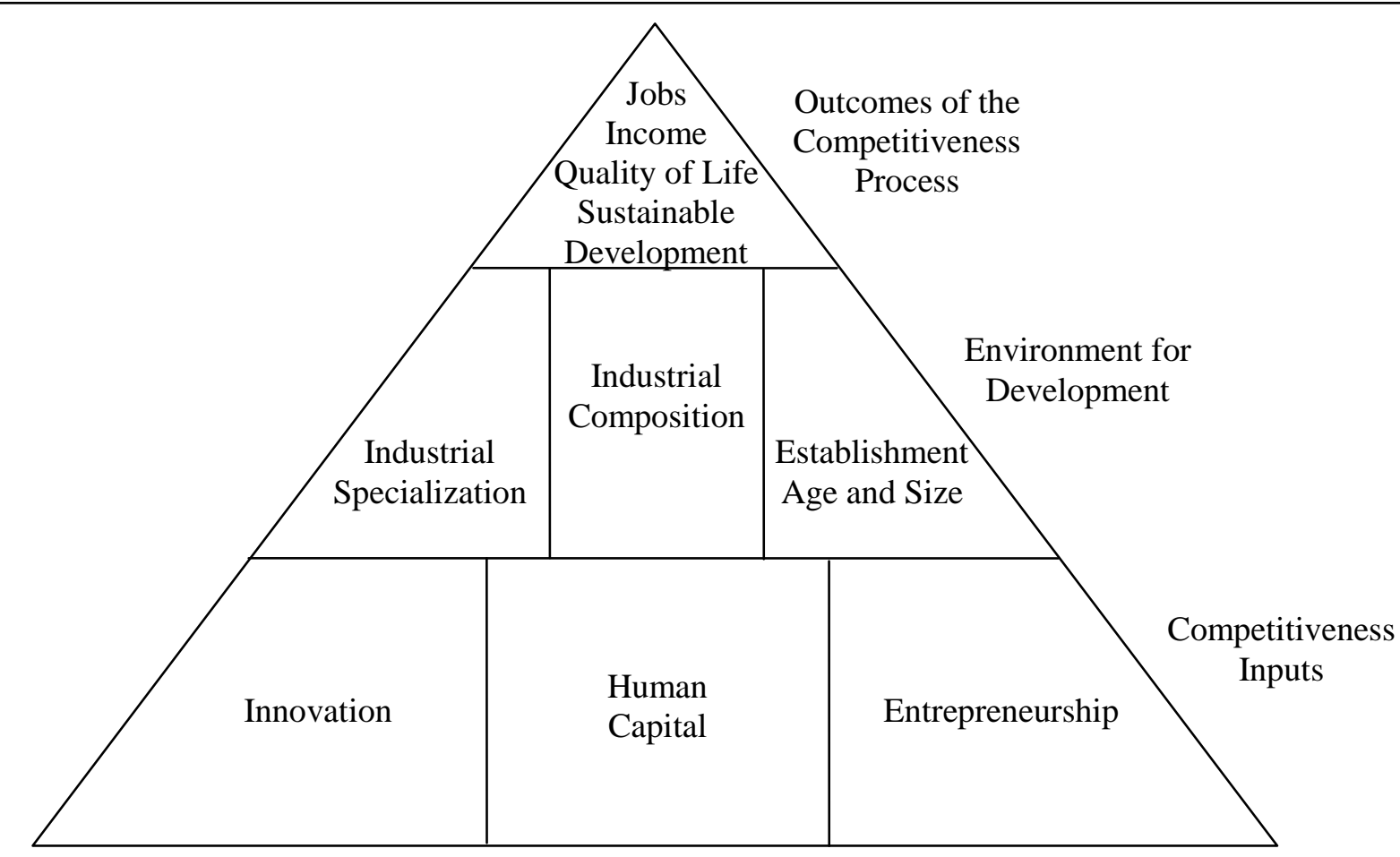

(C) Southern Regional Science Association 2011. 
There is no reason to expect that all of the competitiveness inputs affect all of the economic outcomes with the same magnitude and timing (Atkinson and Gottlieb, 2001; Eberts, Erickcek, and Kleinhenz, 2006; Atkinson and Correa, 2007). For example, labor force education and skills may be more strongly associated with changes in income than changes in population, and entrepreneurial activity may impact growth in employment differently than growth in income. A principal goal of the proposed econometric analysis is to test for the potential differential effects of policy variables on regional outcome growth rates.

\subsubsection{Regional Adjustment Models}

Potential relationships between New Economy inputs to the competitiveness process (innovation, entrepreneurship, and human capital) and outcomes of the process (increases in jobs, residents, and per capita incomes) are investigated by estimating regional adjustment models for Southern metropolitan areas. Regional adjustment models are used frequently in the regional science literature to examine potential determinants of population and employment growth at the census tract, county, and metropolitan levels. ${ }^{3}$ The regional adjustment model selected for this analysis follows the three-equation framework of Deller et al. (2001) and Carruthers and Mulligan (2008) where changes in area population, employment, and per capita income (or wages) are determined simultaneously. Specifically,

$$
\begin{aligned}
& P_{i t}^{*}=\mathrm{f}\left(E_{i t}{ }^{*}, Y_{i t}{ }^{*}, \mathbf{x}_{i t}, e_{i t}\right) \\
& E_{i t}^{*}=\mathrm{f}\left(P_{i t}{ }^{*}, Y_{i t}{ }^{*}, \mathbf{z}_{i t}, e_{i t}\right) \\
& Y_{i t}{ }^{*}=\mathrm{f}\left(P_{i t}{ }^{*}, E_{i t}{ }^{*}, \mathbf{w}_{i t}, e_{i t}\right)
\end{aligned}
$$

where $P_{i t}{ }^{*}, E_{i t}{ }^{*}$, and $Y_{i t}{ }^{*}$ are equilibrium levels of population, employment, and per capita incomes in metropolitan area $i$ at time $t ; \mathbf{x}_{i t}, \mathbf{z}_{i t}$, and $\mathbf{w}_{i t}$ are vectors of initial conditions that affect equilibrium values of the endogenous variables; and $e_{i t}$ are error terms. We do not observe the equilibrium values for the three outcomes of the competitiveness process, but we can measure changes in population, employment, and income over time as functions of the equilibrium values and the speed of adjustment (B) to desired levels. That is:

$$
\begin{aligned}
& P_{t}-P_{t 0}=\mathrm{B}_{p}\left(P^{*}-P_{t 0}\right) \\
& E_{t 1}-E_{t 0}=\mathrm{B}_{e}\left(E^{*}-E_{t 0}\right) \\
& Y_{t 1}-Y_{t 0}=\mathrm{B}_{y}\left(Y^{*}-Y_{t 0}\right)
\end{aligned}
$$

Combining systems of equations (1-3) and (4-6) and expressing in linear terms, Carruthers and Mulligan (2008) propose a system of relationships where the observed rates of change in the outcome measures (population density, employment density, and income/wages) are functions of their initial values, the current values of the remaining outcome measures, and lagged values for initial conditions in the regional economy. The structural form of the regional adjustment model derived by Carruthers and Mulligan is

$$
\begin{aligned}
& \ln \left(\frac{p_{t}}{p_{t-i}}\right)=\alpha_{0 \mathrm{p}}+\alpha_{1 \mathrm{p}} \ln \left(p_{t-i}\right)+\alpha_{2 \mathrm{p}} \ln \left(e_{t}\right)+\alpha_{3 \mathrm{p}} \ln \left(y_{t}\right)+\beta_{\mathrm{p}} \mathbf{x}_{t-i}+\varepsilon_{p t} \\
& \ln \left(\frac{e_{t}}{e_{t-i}}\right)=\alpha_{0 \mathrm{e}}+\alpha_{1 \mathrm{e}} \ln \left(p_{t}\right)+\alpha_{2 \mathrm{e}} \ln \left(e_{t-i}\right)+\alpha_{3 \mathrm{e}} \ln \left(y_{t}\right)+\beta_{\mathrm{e}} \mathbf{x}_{t-i}+\varepsilon_{e t}
\end{aligned}
$$

\footnotetext{
${ }^{3}$ See, for example, Carlino and Mills (1987); Steinnes and Fisher (1974); Clark and Murphy (1996); Henry et al. (1999); Glavac, Vias, and Mulligan (1998); Deller et al. (2001); Henry, Schmitt, and Piguet (2001); Bao, Henry, and Barkley (2004); Carruthers and Mulligan (2008); and Boarnet, Chalermpong, and Geho (2005).
} 


$$
\ln \left(\frac{y_{t}}{y_{t-i}}\right)=\alpha_{0 \mathrm{y}}+\alpha_{1 \mathrm{y}} \ln \left(p_{t}\right)+\alpha_{2 \mathrm{y}} \ln \left(e_{t}\right)+\alpha_{3 \mathrm{y}} \ln \left(y_{t-i}\right)+\beta_{\mathrm{y}} \mathbf{x}_{t-i}+\varepsilon_{y t}
$$

where:

$$
p_{t}=\text { population per square mile at time } t
$$

$p_{t-i}=$ population per square mile at time $t-i$

$e_{t}=$ employment per square mile at time $t$

$e_{t-i}=$ employment per square mile at time $t-i$

$y_{t}=$ per capita income at time $t$

$y_{t-i}=$ per capita income at time $t-i$

$\mathbf{x}_{t-i}=$ vector of policy input and industrial structure variables at time $t-i$

$\varepsilon_{t}=$ error in the estimation of a given outcome at time $t$.

In the above regional adjustment model, the $\alpha_{1 p}, \alpha_{2 e}$, and $\alpha_{3 y}$ coefficients are estimated parameters for the rates of adjustment of population, employment, and income; and regional convergence in these outcomes is represented by negative values for the estimated adjustment parameters.

Regional adjustment models lend themselves to the study of regional competitiveness because the motivating factors in these models are the migration of households and firms in response to perceived economic opportunities and personal preferences. That is, the models assume that both households and firms are free to change locations with households migrating to increase their utility (from both market and nonmarket goods) and firms migrating to enhance their profits (from lower production costs or higher market demand). Thus, insights into the competitiveness of regions may be revealed by the relative ability of regions to attract more people and jobs over time subject to increasing rents and lower quality of life.

A second advantage of examining the competitiveness of metro areas with regional adjustment models is that these models provide insights into whether increases in competitiveness are demand-induced (firms increase employment opportunities to meet increases in demand for exports) or supply-induced (individuals move to a community for nonemployment related reasons and jobs are created to serve the increased population) or both. The original Carlino and Mills (1987) framework presents this relationship between supply- and demand-induced growth as a system of two simultaneous equations where population change over time is a function of employment change over the same period and employment change is a function of coterminous population change. Deller et al. (2001) expand the model to include per capita income as a third equation in the system, and Carruthers and Mulligan (2008) include an equation with average annual wages as an endogenous variable. Deller et al. include the per capita income equation to draw attention to the roles of job quality and local quality of life in the adjustment process. Carruthers and Mulligan, on the other hand, include an endogenous wage variable to account for the equilibrating influences of changing land values that result from changes in population and employment densities. Both Deller et al. and Carruthers and Mulligan hypothesize (1) a negative relationship between changes in population density and current per capita income (wages) and (2) a positive association between changes in employment density and current income (wages), but for slightly different reasons. Deller et al. suggest that high per 
capita incomes reflect high job quality that will attract employers, but higher incomes may discourage population growth if it also reflects high costs of living or low local quality of life. Alternatively, in Carruthers and Mulligan, high wages result in lower population densities as households increase land holdings and bid up land prices. The higher land prices, in turn, encourage businesses to reduce land holdings and increase employment densities. Thus both wages and per capita incomes act as equilibrating forces in the regional adjustment model through their influence on land rents, labor supply and demand, and residential location decisions.

For our analysis of regional competitiveness, we select per capita income as the third endogenous variable in the model. Glaeser, Scheinkman, and Shleifer (1995) also use income in their models of metropolitan growth to account for both job quality and quality of life. Wages capture worker productivity and a large share of workers' standard of living; however, per capita income includes transfers, rents, and dividends in addition to wages and therefore reflects standards of living across a wider population (Alcany, 2003). These additional income sources are important to many metro areas, particularly in the South, that attract retirees.

Finally, this study focuses on short-run changes in regional competitiveness (2000-2006) that are associated with inputs to the competitiveness process. The model does not include quality of life measures, except to the extent that income adjusts to reflect quality of life and human capital is correlated with quality of life. ${ }^{4}$ Nor does the model include infrastructure variables that may influence economic competitiveness (e.g., miles of interstate highway, availability of air transportation). The omitted variables are relatively fixed in the short-run, and these variables are found to be more associated with levels of outcomes than changes in regional outcomes over time. Moreover, the potential for omitted variables bias is reduced since the static factors are cancelled out in the differencing process. ${ }^{5}$

\section{DATA COLLECTION}

\subsection{Study Area}

Data are collected and analyzed for 151 MSAs in the Southern U.S. Census Region. ${ }^{6}$ The states that make up the South are Alabama, Arkansas, Delaware, Florida, Georgia, Kentucky, Louisiana, Maryland, Mississippi, North Carolina, Oklahoma, South Carolina, Tennessee, Texas, Virginia, and West Virginia. Fisher (2005) and Atkinson and Gottlieb (2001) find that the appropriate level of study is the metropolitan area. Metropolitan areas are more cohesive economic units than states, and metropolitan areas share common labor markets and other resources (Haughton and Murg, 2002). Economic conditions can vary dramatically within a state (Fisher, 2005), and the variation of economic factors may be greater within a state than between states (Atkinson, 1990).

\footnotetext{
${ }^{4}$ Mueser and Graves (1995) posit that highly skilled, privileged workers move toward amenity-rich areas.

5 The Carruthers and Mulligan (2008) model includes spatially lagged dependent variables to control for spatial dependence between counties within and surrounding MSAs. However, our research analyzes data at the MSA level rather than the county level. Most southern MSAs are not contiguous or even near enough to facilitate a level of inter-MSA commuting that would interfere with the compensating differential model. In addition, we included a state fixed effects variable to control for state-level influences on metro growth rates.

${ }^{6}$ Wilmington, DE-MD, is actually a metropolitan division within the Philadelphia-Camden-Wilmington, PA-NJ-DE-MD, MSA, and Wilmington is not included in this study. The Washington, DC, MSA is excluded from this study because, as the nation's capital, Washington is dominated by government activity. Washington's economy is fundamentally different from those of other Southern cities.
} 
Each MSA is made up of one or more counties that comprise and surround an urban core. The U.S. Bureau of the Census (Census Bureau) periodically updates the MSAs' boundaries to reflect commuting and population patterns. This study uses the Census Bureau's 2003 MSA definitions that also are used in the 2006 American Community Survey. The MSA boundaries are different for the 2000 and 1990 Censuses. Furthermore, some 2006 MSAs were not considered MSAs in 2000 or 1990 or were combined with another metropolitan area or areas in those years. The 2000 and 1990 MSA data are adjusted using county-level data to conform to the MSA definitions in effect in 2003 and 2006.

Regional adjustment models were originally used to study firm and household migration within closed economic systems, such as the U.S. as a whole (e.g., Carlino and Mills, 1987); however, many recent applications rely on the models to analyze subregions. For example, Carruthers and Mulligan (2008) focus on metropolitan areas, Deller et al. (2001) focus on rural counties in the U.S., and Boarnet, Chalermpong, and Geho (2005) analyze census tracts in Orange County, California. Indeed, given today's global economy, it would be difficult to find a truly closed economic system. Here, the study area is limited to MSAs in the U.S. South because of the availability of special tabulations of data pertaining to establishment age and wages in traded sectors. Estimated coefficients must be interpreted as changes relative to other Southern MSAs.

The U.S. South, as a whole, is behind the nation in the transition to New Economy activities. For example, manufacturing employment, as a share of total employment, is higher in the South than the nation as a whole. In addition, educational attainment levels in the South continue to lag the nation. Only one state in the South (Virginia) has more college graduates (as a share of the adult population) than the national average, and only two Southern states (Oklahoma and Virginia) have a greater share of high school graduates than the nation's average. Yet, numerous metro areas in the South (e.g., Austin, Texas; Raleigh-Durham, North Carolina; Huntsville, Alabama; Lexington, Kentucky) have high levels of educational attachment, knowledge workers, innovative activity, and entrepreneurial and small business development. The diversity of economic development histories and experiences in the South provides a good study area for assessing the roles of New Economy policies on regional competitiveness.

\subsection{Variable Selection and Measures}

In this study, representatives of 25 variables previously associated with the competitive environment and industrial structure are used to benchmark rates of change in population, employment, and per capita income in the MSAs of U.S. South. The selected variables are based on findings of earlier studies of the determinants of regional growth and measures used in reports that rank or grade regional competitiveness. Data are collected for each of the Southern MSAs for 1990, 2000, and 2006. In some cases, data are not available for a particular year, and data from the nearest year are used in their place. For example, the number of Ph.D. students in science and engineering fields is not available for the base year 1990, thus 1994 (earliest available) data are used. For discussion purposes, the variables are grouped according to whether the measure is selected to represent regional competitiveness outcomes, policy inputs, or industrial structure. A listing of the selected measures and data sources are provided in Table 1, and descriptive statistics for the variables are provided in Table 2.

\footnotetext{
${ }^{7}$ Enid, Oklahoma, was a metropolitan statistical area in 2000, then became a micropolitan statistical area with the introduction of that designation in 2003. Since it was not a metropolitan statistical area in 2006, it was excluded from this study.
} 


\subsubsection{Outcome Variables for Regional Competitiveness}

Population. Many studies use population growth to reflect regional competitiveness (e.g., Carlino and Mills, 1987; Glaeser, Scheinkman, and Shleifer, 1995; Carruthers and Mulligan, 2008). ${ }^{8}$ If competitive regions are defined as "places where both companies and people want to locate and invest in" (Kitson, Martin, and Tyler, 2004, p. 997), then by definition, competitive regions have growing populations. Population growth rates are hypothesized to display convergence through a negative association with lagged (base year) population levels. Alternatively, a positive association with regional employment and per capita income is expected if people migrate to take advantage of job opportunities (Carruthers and Mulligan, 2008; Glaeser, Schinkman, and Shleifer, 1995).

Employment. Eberts, Erickcek, and Kleinhenz (2006) note that population growth can also be associated with urban sprawl; therefore, population growth by itself is not a good measure of regional competitiveness. Following Carlino and Mills (1987), Carruthers and Mulligan (2008), Eberts, Erickcek, and Kleinhenz (2006), and the Corporation for Enterprise Development (2007), the metro growth rate of employment is added as an outcome measure of competitiveness. Employment growth reflects the attractiveness of the regions to businesses and the growth of existing area businesses. Measuring population and employment as densities demonstrates spatial intensity and also allows more ready comparisons across MSAs of varying populations and land areas.

Per Capita Income. The third outcome measure is per capita income, a proxy measure for productivity and the quality of life aspect of competitiveness (Eberts, Erickcek, and Kleinhenz, 2006; Glaeser, Scheinkman, and Shleifer, 1995). The growth rate of per capita income reflects the effects of labor supply and demand on wages, and it is expected to be negatively related to population (potential labor supply) but positively related to employment (labor demand) (Carruthers and Mulligan, 2008). ${ }^{9}$

\subsubsection{Input Variables for Regional Competitiveness}

Innovation. Innovation is widely regarded as a driver of economic growth and competitiveness (Acs, 2002; Audretsch, 2000; Camp, 2005). Measures of innovation include graduate students in science and engineering per 10,000 residents; $\mathrm{PhD}$ degrees awarded in science and engineering per 10,000 residents; academic research and development (R\&D) funding per capita; college and graduate school enrollment per 10,000 residents; the percent of the population ages 25 or older with a graduate or professional degree; and the percent of employment in computer, science, and engineering occupations (Atkinson and Gottlieb, 2001; Eberts, Erickcek, and Kleinhenz, 2006; Gardiner, 2003; Tuerck et al., 2007). The innovation inputs are expected to be positively associated with population, employment, and income growth.

\footnotetext{
${ }^{8}$ The model actually includes population and employment per square mile. However, the growth in the densities is identical to the growth $n$ population and employment because the land area of the counties is held constant over the study period. Thus, the denominators cancel, leaving the growth in the outcome variables.

9 Attempts to account for cost of living differences across MSAs using American Chamber of Commerce Researchers Association $(1990,2000,2006)$ data produced less reliable results due to inconsistencies in data availability and measurement across years.
}

(C) Southern Regional Science Association 2011. 
TABLE 1. Competitiveness Variables, Years, and Data Sources

\begin{tabular}{|c|c|c|c|}
\hline Element & Variable & Year & Data Source \\
\hline \multirow{9}{*}{ Outcomes } & \multirow{3}{*}{$\begin{array}{l}\text { Growth rate of population } \\
\text { (and lagged population) }\end{array}$} & 2006 & $\begin{array}{l}\text { U.S. Census Bureau, 2007, } 2006 \text { American } \\
\text { Community Survey, Table B01003 }\end{array}$ \\
\hline & & 2000 & $\begin{array}{l}\text { U.S. Census Bureau, 2002, } 2000 \text { Decennial } \\
\text { Census, Table P1 }\end{array}$ \\
\hline & & 1990 & $\begin{array}{l}\text { U.S. Census Bureau, 1993, } 1990 \text { Decennial } \\
\text { Census, Table P001 }\end{array}$ \\
\hline & \multirow{3}{*}{$\begin{array}{l}\text { Growth rate of employment } \\
\text { (and lagged employment) }\end{array}$} & 2006 & $\begin{array}{l}\text { U.S. Census Bureau, 2008, County Business } \\
\text { Patterns }\end{array}$ \\
\hline & & 2000 & $\begin{array}{l}\text { U.S. Census Bureau, 2008, County Business } \\
\text { Patterns }\end{array}$ \\
\hline & & 1990 & $\begin{array}{l}\text { U.S. Census Bureau, 2008, County Business } \\
\text { Patterns }\end{array}$ \\
\hline & \multirow{3}{*}{$\begin{array}{l}\text { Growth rate of per capita income } \\
\text { (and lagged per capita income) }\end{array}$} & 2006 & $\begin{array}{l}\text { U.S. Census Bureau, 2007, } 2006 \text { American } \\
\text { Community Survey, Table B19301 }\end{array}$ \\
\hline & & 2000 & $\begin{array}{l}\text { U.S. Census Bureau, 2002, } 2000 \text { Decennial } \\
\text { Census, Table P82 }\end{array}$ \\
\hline & & 1990 & $\begin{array}{l}\text { U.S. Census Bureau, 1993, } 1990 \text { Decennial } \\
\text { Census, Table P114A }\end{array}$ \\
\hline \multicolumn{4}{|c|}{ Measures of the Competitiveness Policy Inputs: } \\
\hline \multirow{22}{*}{ Innovation } & \multirow{2}{*}{$\begin{array}{l}\text { College and graduate school enrollment per } \\
10,000 \text { population }\end{array}$} & 2000 & $\begin{array}{l}\text { U.S. Census Bureau, 2002, } 2000 \text { Decennial } \\
\text { Census, Table P36 }\end{array}$ \\
\hline & & 1990 & $\begin{array}{l}\text { U.S. Census Bureau, 1993, } 1990 \text { Decennial } \\
\text { Census, Table P054 }\end{array}$ \\
\hline & \multirow{2}{*}{$\begin{array}{l}\text { Percent of population ages } 25+\text { with an advanced } \\
\text { degree }\end{array}$} & 2000 & $\begin{array}{l}\text { U.S. Census Bureau, 2002, } 2000 \text { Decennial } \\
\text { Census, Table P37 }\end{array}$ \\
\hline & & 1990 & $\begin{array}{l}\text { U.S. Census Bureau, 1993, } 1990 \text { Decennial } \\
\text { Census, Table P054 }\end{array}$ \\
\hline & \multirow{2}{*}{$\begin{array}{l}\text { Graduate students in science and engineering per } \\
10,000 \text { residents }\end{array}$} & 2000 & National Science Foundation, 2002b \\
\hline & & 1994 & National Science Foundation, 1996 \\
\hline & \multirow{2}{*}{$\begin{array}{l}\text { Science and engineering PhD's per 10,000 } \\
\text { residents }\end{array}$} & 2000 & National Science Foundation, 2001 \\
\hline & & 1994 & National Science Foundation, 1995 \\
\hline & \multirow{2}{*}{ Academic R\&D spending per capita } & 2000 & National Science Foundation, 2002a \\
\hline & & 1990 & National Science Foundation, 1999 \\
\hline & \multirow{2}{*}{$\begin{array}{l}\text { Percent of employment in computer, science, and } \\
\text { engineering occupations }\end{array}$} & 2000 & $\begin{array}{l}\text { U.S. Census Bureau, 2002, } 2000 \text { Decennial } \\
\text { Census, Table P50 }\end{array}$ \\
\hline & & 1990 & $\begin{array}{l}\text { U.S. Census Bureau, 1993, } 1990 \text { Decennial } \\
\text { Census, Table P078 }\end{array}$ \\
\hline & \multirow{2}{*}{$\begin{array}{l}\text { Percent of population ages } 25+\text { with less than high } \\
\text { a school diploma }\end{array}$} & 2000 & $\begin{array}{l}\text { U.S. Census Bureau, 2002, } 2000 \text { Decennial } \\
\text { Census, Table P37 }\end{array}$ \\
\hline & & 1990 & $\begin{array}{l}\text { U.S. Census Bureau, 1993, } 1990 \text { Decennial } \\
\text { Census, Table P054 }\end{array}$ \\
\hline & \multirow{2}{*}{ Percent of population of working age (25-64) } & 2000 & $\begin{array}{l}\text { U.S. Census Bureau, 2002, } 2000 \text { Decennial } \\
\text { Census, Table P8 }\end{array}$ \\
\hline & & 1990 & $\begin{array}{l}\text { U.S. Census Bureau, 1993, } 1990 \text { Decennial } \\
\text { Census, Table P013 }\end{array}$ \\
\hline & \multirow{2}{*}{ Labor force participation rate } & 2000 & $\begin{array}{l}\text { U.S. Census Bureau, 2002, } 2000 \text { Decennial } \\
\text { Census, Table P43 }\end{array}$ \\
\hline & & 1990 & $\begin{array}{l}\text { U.S. Census Bureau, 1993, } 1990 \text { Decennial } \\
\text { Census, Table P070 }\end{array}$ \\
\hline & \multirow{2}{*}{ Employment rate (employed/labor force) } & 2000 & $\begin{array}{l}\text { U.S. Census Bureau, 2002, } 2000 \text { Decennial } \\
\text { Census, Table P43 }\end{array}$ \\
\hline & & 1990 & $\begin{array}{l}\text { U.S. Census Bureau, 1993, } 1990 \text { Decennial } \\
\text { Census, Table P070 }\end{array}$ \\
\hline & \multirow{2}{*}{ Out-of-poverty rate } & 2000 & $\begin{array}{l}\text { U.S. Census Bureau, 2002, } 2000 \text { Decennial } \\
\text { Census, Table P87 }\end{array}$ \\
\hline & & 1990 & $\begin{array}{l}\text { U.S. Census Bureau, 1993, } 1990 \text { Decennial } \\
\text { Census, Table P117 }\end{array}$ \\
\hline
\end{tabular}

(Continued) 
TABLE 1. Competitiveness Variables, Years, and Data Sources (Cont'd)

\begin{tabular}{|c|c|c|c|}
\hline \multicolumn{4}{|c|}{ Measures of the Competitiveness Policy Inputs, Continued: } \\
\hline Element & Variable & Year & Data Source \\
\hline & \multirow{2}{*}{ Percent of population who speak English well } & 2000 & $\begin{array}{l}\text { U.S. Census Bureau, 2002, } 2000 \text { Decennial } \\
\text { Census, Table P19 }\end{array}$ \\
\hline & & 1990 & $\begin{array}{l}\text { U.S. Census Bureau, 1993, } 1990 \text { Decennial } \\
\text { Census, Table P028 }\end{array}$ \\
\hline & \multirow{2}{*}{ Percent of population that is a racial minority } & 2000 & $\begin{array}{l}\text { U.S. Census Bureau, 2002, } 2000 \text { Decennial } \\
\text { Census, Table P6 }\end{array}$ \\
\hline & & 1990 & $\begin{array}{l}\text { U.S. Census Bureau, 1993, } 1990 \text { Decennial } \\
\text { Census, Table P008 }\end{array}$ \\
\hline & \multirow{2}{*}{$\begin{array}{l}\text { Percent of employment in computer, science, and } \\
\text { engineering occupations }\end{array}$} & 2000 & $\begin{array}{l}\text { U.S. Census Bureau, 2002, } 2000 \text { Decennial } \\
\text { Census, Table P50 }\end{array}$ \\
\hline & & 1990 & $\begin{array}{l}\text { U.S. Census Bureau, 1993, } 1990 \text { Decennial } \\
\text { Census, Table P078 }\end{array}$ \\
\hline & \multirow{2}{*}{$\begin{array}{l}\text { Percent of employment in management, } \\
\text { business/operations, finance, computers, math, } \\
\text { architecture, engineering, sciences, law, } \\
\text { education, healthcare, arts, design, entertainment, } \\
\text { media, and high-end sales occupations }\end{array}$} & 2000 & $\begin{array}{l}\text { U.S. Census Bureau, 2002, } 2000 \text { Decennial } \\
\text { Census, Table P50 }\end{array}$ \\
\hline & & 1990 & $\begin{array}{l}\text { U.S. Census Bureau, 1993, } 1990 \text { Decennial } \\
\text { Census, Table P078 }\end{array}$ \\
\hline & \multirow{2}{*}{$\begin{array}{l}\text { Percent of employment in professional, scientific, } \\
\text { and technical services industries }\end{array}$} & 2000 & $\begin{array}{l}\text { U.S. Census Bureau, 2002, } 2000 \text { Decennial } \\
\text { Census, Table P49 }\end{array}$ \\
\hline & & 1990 & $\begin{array}{l}\text { U.S. Census Bureau, 1993, } 1990 \text { Decennial } \\
\text { Census, Table P077 }\end{array}$ \\
\hline & \multirow{2}{*}{$\begin{array}{l}\text { Percent of population ages } 25+\text { with a bachelor's } \\
\text { degree }\end{array}$} & 2000 & $\begin{array}{l}\text { U.S. Census Bureau, 2002, } 2000 \text { Decennial } \\
\text { Census, Table P37 }\end{array}$ \\
\hline & & 1990 & $\begin{array}{l}\text { U.S. Census Bureau, 1993, } 1990 \text { Decennial } \\
\text { Census, Table P054 }\end{array}$ \\
\hline \multicolumn{4}{|c|}{ Measures of the Environment for Development: } \\
\hline Element & Variable & Year & Data Source \\
\hline \multirow{6}{*}{ Entrepreneurship } & \multirow{2}{*}{ Number of proprietors per capita } & 2000 & $\begin{array}{l}\text { U.S. BEA, 2008, Local Area Personal } \\
\text { Income, Table CA030 }\end{array}$ \\
\hline & & 1990 & $\begin{array}{l}\text { U.S. BEA, 2008, Local Area Personal } \\
\text { Income, Table CA030 }\end{array}$ \\
\hline & \multirow{2}{*}{ Proprietors' income as a share of total earnings } & 2000 & $\begin{array}{l}\text { U.S. BEA, 2008, Local Area Personal } \\
\text { Income, Table CA05 }\end{array}$ \\
\hline & & 1990 & $\begin{array}{l}\text { U.S. BEA, 2008, Local Area Personal } \\
\text { Income, Table CA05 }\end{array}$ \\
\hline & \multirow{2}{*}{$\begin{array}{l}\text { Business churning ([births }+ \text { deaths }] / \text { initial } \\
\text { establishments) }\end{array}$} & 2000 & $\begin{array}{l}\text { U.S. Census Bureau, 2001, 2000-2001 } \\
\text { Statistics of U.S. Businesses }\end{array}$ \\
\hline & & 1998 & $\begin{array}{l}\text { U.S. Census Bureau, 1999, 1998-1999 } \\
\text { Statistics of U.S. Businesses }\end{array}$ \\
\hline \multirow{6}{*}{$\begin{array}{l}\text { Establishment age } \\
\text { and size }\end{array}$} & \multirow{2}{*}{ Percent of establishments fewer than 5 years old } & 2000 & $\begin{array}{l}\text { U.S. Census Bureau, 2008, Special } \\
\text { Tabulation }\end{array}$ \\
\hline & & 1990 & $\begin{array}{l}\text { U.S. Census Bureau, 2008, Special } \\
\text { Tabulation }\end{array}$ \\
\hline & \multirow{2}{*}{ Establishments per employee } & 2000 & $\begin{array}{l}\text { U.S. Census Bureau, 2008, County Business } \\
\text { Patterns }\end{array}$ \\
\hline & & 1990 & $\begin{array}{l}\text { U.S. Census Bureau, 2008, County Business } \\
\text { Patterns }\end{array}$ \\
\hline & \multirow{2}{*}{$\begin{array}{l}\text { Percent of establishments with fewer than } 20 \\
\text { employees }\end{array}$} & 2000 & $\begin{array}{l}\text { U.S. Census Bureau, 2008, County Business } \\
\text { Patterns }\end{array}$ \\
\hline & & 1990 & $\begin{array}{l}\text { U.S. Census Bureau, 2008, County Business } \\
\text { Patterns }\end{array}$ \\
\hline \multirow{2}{*}{$\begin{array}{l}\text { Industrial } \\
\text { specialization }\end{array}$} & \multirow{2}{*}{ Employment specialization index } & 2000 & $\begin{array}{l}\text { U.S. Census Bureau, 2002, } 2000 \text { Decennial } \\
\text { Census, Table P49 }\end{array}$ \\
\hline & & 1990 & $\begin{array}{l}\text { U.S. Census Bureau, 1993, } 1990 \text { Decennial } \\
\text { Census, Table P077 }\end{array}$ \\
\hline \multirow{4}{*}{$\begin{array}{l}\text { Industrial } \\
\text { composition }\end{array}$} & \multirow{2}{*}{ Relative wage of occupations in traded industries } & 2000 & Harvard Business School, 2008 \\
\hline & & 1990 & Harvard Business School, 2008 \\
\hline & \multirow{2}{*}{ Percent of employment in manufacturing sectors } & 2000 & $\begin{array}{l}\text { U.S. Census Bureau, 2002, } 2000 \text { Decennial } \\
\text { Census, Table P49 }\end{array}$ \\
\hline & & 1990 & $\begin{array}{l}\text { U.S. Census Bureau, 1993, } 1990 \text { Decennial } \\
\text { Census, Table P077 }\end{array}$ \\
\hline
\end{tabular}


TABLE 2. Descriptive Statistics for Study Variables

\begin{tabular}{|c|c|c|c|c|c|}
\hline Variable & Mean & Med. & Min. & Max. & S.D. \\
\hline Growth rate of population, $2000-2006$ & 0.0732 & 0.0667 & -0.2846 & 0.5033 & 0.0803 \\
\hline Growth rate of employment, 2000-2006 & 0.0909 & 0.0764 & -0.2277 & 0.7120 & 0.1020 \\
\hline Growth rate of per capita income, $2000-2006$ & 0.1543 & 0.1537 & -0.0185 & 0.3453 & 0.0536 \\
\hline Population, 2006 & $557,768.33$ & $240,450.50$ & 71,667 & $5,982,787$ & $954,544.68$ \\
\hline Employment, 2006 & $261,731.99$ & $116,224.00$ & 26,745 & $2,977,990$ & $465,670.42$ \\
\hline Per capita income, 2006 & $22,076.24$ & $21,496.50$ & 11,919 & 34,650 & $3,580.58$ \\
\hline Population, 2000 & $507,829.85$ & $227,569.00$ & 49,832 & $5,161,544$ & $845,857.62$ \\
\hline Employment, 2000 & $233,344.03$ & $101,289.00$ & 18,815 & $2,550,873$ & $400,279.30$ \\
\hline Per capita income, 2000 & $18,892.78$ & $18,403.73$ & 9,899 & 31,195 & $2,921.95$ \\
\hline Population, 1990 & $425,571.78$ & $192,018.50$ & 28,701 & $4,056,100$ & $670,269.79$ \\
\hline Employment, 1990 & $196,796.60$ & $81,021.50$ & 10,542 & $2,055,606$ & $328,981.62$ \\
\hline Per capita income, 1990 & $12,321.36$ & $12,044.43$ & $6,630.00$ & $21,386.00$ & $2,008.43$ \\
\hline Graduate students in science and engineering per 10,000 & 19.06 & 4.76 & 0.00 & 322.39 & 44.99 \\
\hline Science and engineering $\mathrm{PhD}$ 's per 10,000 residents & 1.15 & 0.00 & 0.00 & 28.74 & 3.61 \\
\hline Academic R\&D spending per capita & 73.93 & 0.0000 & 0.0000 & $1,806.65$ & 224.83 \\
\hline College and graduate school enrollment per 10,000 residents & 719.69 & 630.51 & 288.89 & $2,707.92$ & 392.86 \\
\hline Percent of population ages $25+$ with an advanced degree & $6.03 \%$ & $5.52 \%$ & $2.62 \%$ & $16.19 \%$ & $2.32 \%$ \\
\hline Percent of employment in computer, science, and engineering occs & $4.64 \%$ & $4.55 \%$ & $2.77 \%$ & $7.79 \%$ & $0.87 \%$ \\
\hline Percent of population ages $25+$ with less than a high school diploma & $28.76 \%$ & $27.96 \%$ & $16.23 \%$ & $53.39 \%$ & $6.31 \%$ \\
\hline Percent of population of working age (25-64) & $50.12 \%$ & $50.16 \%$ & $41.15 \%$ & $55.49 \%$ & $2.67 \%$ \\
\hline Labor force participation rate & $48.80 \%$ & $49.17 \%$ & $36.36 \%$ & $61.46 \%$ & $4.23 \%$ \\
\hline Employment rate (employed/labor force) & $93.72 \%$ & $93.84 \%$ & $85.69 \%$ & $96.77 \%$ & $1.77 \%$ \\
\hline Out-of-poverty rate & $83.95 \%$ & $84.76 \%$ & $58.12 \%$ & $92.45 \%$ & $5.50 \%$ \\
\hline Percent of population who speak English well & $98.47 \%$ & $99.40 \%$ & $77.56 \%$ & $99.82 \%$ & $3.30 \%$ \\
\hline Percent of population that is a racial minority & $20.44 \%$ & $20.66 \%$ & $1.60 \%$ & $45.72 \%$ & $11.00 \%$ \\
\hline $\begin{array}{l}\text { Percent of employment in management, business/operations, finance, computers } \\
\text { math, architecture, engineering, sciences, law, education, healthcare, arts, } \\
\text { design, entertainment, media, and high-end sales occupations }\end{array}$ & $37.93 \%$ & $37.88 \%$ & $27.05 \%$ & $49.55 \%$ & $4.24 \%$ \\
\hline Percent of employment in professional, scientific, and technical service industries & $4.06 \%$ & $4.03 \%$ & $2.39 \%$ & $5.90 \%$ & $0.68 \%$ \\
\hline Percent of population ages $25+$ with a bachelor's degree & $11.22 \%$ & $10.67 \%$ & $5.99 \%$ & $20.72 \%$ & $3.04 \%$ \\
\hline Number of proprietors per capita & 0.0729 & 0.0694 & 0.0223 & 0.1767 & 0.0213 \\
\hline Proprietors' income as a share of total earnings & 0.0867 & 0.0842 & 0.0303 & 0.2308 & 0.0291 \\
\hline Business churning ([births + deaths $] /$ initial establishments) & 0.2226 & 0.2200 & 0.1550 & 0.3265 & 0.0278 \\
\hline Percent of employment fewer than 5 years old & $44.18 \%$ & $43.56 \%$ & $33.82 \%$ & $60.79 \%$ & $4.68 \%$ \\
\hline Establishments per employee & 0.0712 & 0.0693 & 0.0470 & 0.1052 & 0.0125 \\
\hline Percent of establishments with fewer than 20 employees & $87.03 \%$ & $86.92 \%$ & $82.84 \%$ & $91.79 \%$ & $1.60 \%$ \\
\hline Employment specialization index & 0.2661 & 0.2514 & 0.0809 & 0.7295 & 0.0947 \\
\hline Relative wage of occupations in traded industries & 0.7387 & 0.7257 & 0.3808 & 1.2957 & 0.1402 \\
\hline Percent of employment in manufacturing sectors & $17.89 \%$ & $16.18 \%$ & $4.88 \%$ & $48.35 \%$ & $8.19 \%$ \\
\hline
\end{tabular}

(c) Southern Regional Science Association 2011.

ISSN 1553-0892

SRSA, 1601 University Avenue, PO Box 6025, Morgantown, West Virginia 26506-6025, USA. 
Innovative activity and the commercialization of resulting innovations are represented in earlier studies by patents per capita (Atkinson and Gottlieb, 2001; Eberts Erickcek, and Kleinhenz, 2006; CFED, 2007) and local venture capital investments (Atkinson and Correa, 2007; Austrian, Lendel, and Yamoah, 2007; CFED, 2007; Tuerck et al., 2007). This study excludes patent data because it represents an intermediate output of the innovation process rather than an input to the process. Venture capital investments are not included because reliable data are not available for Southern metropolitan areas for 1990.

Human Capital. In the New Economy, skilled labor is often more important to firms than is inexpensive labor (Malecki, 2004), and earlier studies recognize the importance of abundant skilled labor in promoting economic growth (Eberts, Erickcek, and Kleinhenz, 2006; Tuerck et al., 2007, 2008). The labor skills and employability variables selected for this study include the percent of the population ages 25 and older with less than a high-school diploma; the percent of the population ages 25-64 (working age); the labor-force participation rate; the employment rate; the percent of the population who are not in poverty; the percent of the population who speak English well; and the percent of the population that is considered a racial minority (defined as non-white).

A critical component of a region's human capital is highly educated people in creative occupations (Florida, 2002a, 2002b). Florida proposes that the "knowledge workers" or "creative class" stimulate economic growth by starting businesses and attracting other highskilled workers. Drennan $(1999,2002)$ finds that regions specialized in information services (that include many knowledge workers) have higher education attainment than did other regions and are associated with greater economic growth. Variables in the knowledge workers grouping include the percent of employment in computer, science, and engineering occupations (discussed with the innovation inputs); the percent of people employed in management, business/operations, finance, computers, math, architecture, engineering, sciences, law, education, healthcare, arts, design, entertainment, media, and high-end sales occupations; the percent of employment in professional, scientific, and technical industries; and the percent of the population older than age 25 with a bachelor's degree. Knowledge workers are expected to have a positive effect on the growth rates of population, employment, and per capita income.

Entrepreneurship. Entrepreneurship is an important component of economic development because local business owners are more likely to build supply linkages with other local businesses and to spend profits locally, thus enhancing multiplier effects (Barkley, 2001; Markusen, 1996). Small businesses also are an important source of employment opportunities and job growth. Entrepreneurship has multiple dimensions that are represented by three variables: the number of proprietors per capita, the ratio of proprietors' income to total earnings in the MSA, and a measure of business churning to capture local forces of Schumpeter's (1942) creative destruction (establishment births and death as a percent of total establishments). Entrepreneurship is expected to be associated with higher employment and per capita income growth rates, but the impact on population growth is uncertain. 


\subsubsection{Regional Industrial Structure and Legacy Variables}

Establishment Age and Size. Earlier studies indicate that regions with a relatively large number of young and small businesses have more rapid growth rates in employment and income (Steinle, 1992; Faberman, 2007; Davis, Haltiwanger, and Jarmin, 2008). In addition, Shaffer (2002) and Glaeser et al. (1992) find that regional economic growth is stronger when employment is spread across a large number of firms rather than concentrated in a few large establishments. The selected measure for establishment age is the percent of establishments that are less than five years old. Small establishments are estimated by the percent of establishments with 20 or fewer employees and the number of establishments per employee (Glaeser's measure of competitiveness).

Industrial Specialization. Industrial specialization may indicate the presence of industry clusters that provide agglomeration economies in terms of localization economies, specialized labor pools, and knowledge spillovers. Alternatively, industrial diversity may help regions to dampen business cycles, adapt to long-term shifts in economic activity, and take advantage of urbanization economies (Barkley and Henry, 2001; CFED, 2003; Dissart, 2003; Malecki, 2004).

In this study, industrial specialization $\left(S P E C_{r}\right)$ is calculated using Krugman's (1991) index of employment specialization:

$$
S P E C_{r}=\sum_{i=1}^{n}\left|\frac{E M P_{i, r}}{E M P_{r}}-\frac{E M P_{i, U S}}{E M P_{r U S}}\right|,
$$

where $E M P_{i, r}$ and $E M P_{r}$ are industry $i$ employment in region $r$ and total employment in region $r$, respectively, and $E M P_{i, U . S .}$ and $E M P_{U . S .}$ are U.S. employment in industry $i$ and total employment. Industry employment is measured at the two-digit SIC level for 1990. There are no ex ante expectations for relationships between industrial specialization and the growth rates of population, employment, or per capita income.

Industrial Composition. Industrial composition describes the types of industries that traditionally sustained the region's economy, and the industry composition measures selected for this study are the percent of employment in manufacturing and the relative wage of the region's "traded industries." The relative wage within traded industries compares the average wages in a region's traded (export) industries to the average wages in those same industries in the US. Data on average wages in traded sectors are provided by the Cluster Mapping Project at the Harvard Business School (2008).

The relative wage is a proxy measure for regional productivity after controlling for industry mix (DeVol, Bedroussian, and Kim, 2007). In addition, Porter (2003) recommends a focus on the traded sector because these basic industries play a considerable role in driving wages and, to a lesser degree, employment throughout all industries in the region.

Each region's relative wage within its traded industries (RelativeIndWage ${ }_{r}$ ) is estimated as

$$
\text { (11) RelativeIndWage }{ }_{r}=\sum_{i}\left(\frac{E M P_{i, r}}{E M P_{r}} \times \frac{W_{i, r}}{W_{i, U S}}\right) \text {, }
$$

where $E M P_{i, r}$ is employment in traded industry $i$ in region $r, E M P_{r}$ is employment in all traded industries in the region, $W_{i, r}$ is the average wage in industry $i$ in region $r$, and $W_{i, U . S}$ is the U.S. average wage for industry $i$. If RelativeIndWage $r$ is greater than one, then region $r$ 's average wage in its traded industries is higher than the U.S. average wage in those same industries. 
Regions with higher relative industry wages are hypothesized to have higher labor productivity in their traded sectors. Therefore, high relative wages are predicted to be associated with higher growth rates of population, employment, and per capita income. Conversely, high relative levels of manufacturing activity are expected to be associated with slower growth rates for each of the three outcomes in the post-industrial New Economy. ${ }^{10}$

State Fixed Effects. State tax laws and other policies regarding industrial development (e.g., tax relief incentives, site preparation, and state educational investments) also affect growth in population, employment, and income (Fisher, 2005). The South is generally regarded as having pro-business tax and labor structures (Rork, 2005). However, states differ widely in their laws and tax structures. For example, Georgia has both a corporate income tax and a personal income tax (Graham, 2011) while Texas has a franchise tax on corporations but no personal income tax (Combs, 2009). States also differ in their educational programs and quality of life attributes, state characteristics of importance to current and potential residents. The use of state dummy variables accounts for these differences and other fixed effects among states.

\subsection{Factor Analysis}

The variables selected to represent competitiveness inputs and industrial structure are measured in per capita terms to prevent the values of the large cities from overwhelming data from the smaller cities. Policy and industry structure variables are standardized to a mean of zero and a standard deviation of one. Standardization facilitates the combination of variables with different measurement scales (e.g., dollars per person and establishments per employee) and prevents larger absolute values from dominating the analysis.

Several of the selected variables measure similar metropolitan area characteristics and therefore, some of the variables are highly correlated. Factor analysis is used to categorize variables into groupings, each with a common underlying characteristic called a factor. The variable most strongly associated with the factor is selected to represent the variables in the grouping. Selecting a single measure from among the correlated variables reduces the instability and imprecision caused by multicollinearity (Greene, 2003; Intriligator, 1978). ${ }^{11}$

The factor analysis (Appendix) identifies three factor groupings that reflect inputs to the competitiveness process (referred to as innovation inputs, knowledge workers, and labor employability), two groups that represent industry structure (industrial specialization and industrial composition), and three groups that represent a combination of industrial structure and entrepreneurial environment (proprietors, establishment age, and establishment size). ${ }^{12}$ The innovation inputs factor includes five measures of academic R\&D activity (e.g., R\&D expenditures and students in science and engineering) and our proxy measure for industrial R\&D (percent of employment in computer, science, and engineering occupations). The number of

\footnotetext{
${ }^{10}$ The relative industry wage measure may also reflect cost of living differences among the metro areas. No adjustments for cost of living differences are included in the relative wage measure because cost of living deflators are not available for all the metro areas.

${ }^{11}$ A composite measure made up of the variables with a common factor is used in an alternative version of the model. Results from these estimations are available by contacting the authors.

12 The principal-factor method is used to group variables according to their squared correlation coefficients. Eight factors are identified through evaluation of eigenvalues and scree tests in Stata (StataCorp, 2005). A minimum eigenvalue of one provides factors that are easy to interpret, fit well with economic theory and previous research results, and are reasonably invariant to changes in variables and structure. The factor pattern matrix is then rotated orthogonally (varimax rotation) to more easily identify variables with a common factor and to facilitate interpretation of the factors (Hatcher, 1994; Kim and Mueller, 1978).
} 
science and engineering graduate students per 10,000 residents loads most strongly on the factor and is selected to represent the innovation inputs grouping in the model.

The knowledge workers factor has positive loadings for the percent of the adult population with a bachelor's degree and the percent of employment in technical occupations, creative class, and "high tech" industries. The factor has negative loadings for employment in manufacturing and population without a high school degree. The negative loadings for measures of manufacturing and high school dropouts on this factor are consistent with earlier research indicating that the more highly educated and skilled workers avoid locations with significant manufacturing activity or generally low levels of educational attainment for the labor force (Wheeler, 2006). The share of employment in management, business, and professional occupations (a broad definition of knowledge workers [Huggins, 2003; Gabe, 2006]) represents the variable grouping in the model.

The labor employability factor is a grouping of six measures that reflect the general conditions of the labor market and quality of the area labor force (share of population that is of working age, employment and labor force participation rates, share of population who speak English well, and percent who are not in poverty). The share of the labor force without a high school degree is negatively related to the other variables in the labor employability factor. The employment rate (employed/labor force) is selected as an appropriate representative of the labor employability factor.

The proprietors grouping includes positive loadings for two measures of the importance of proprietors in the local economy (number of proprietors and proprietors' income as a share of total earnings) and a negative loading for the percent of population that is a racial minority. The negative association between measures of proprietor activity and racial minorities is consistent with the findings of Fairlie and Robb (2008) and Robinson, Dassie, and Christy (2004) that minorities often lack access to the human, financial, and social capital beneficial to operating a small business. The number of proprietors per capita is selected to represent this grouping.

Finally, the factor analysis allocates the five selected measures of industrial structure (plus one measure of entrepreneurial environment) into four factors. The establishment age factor includes business churning (births plus deaths as a share of total establishments) and the percent of establishments that were relatively new (less than five years old). The factor is represented in the model by the percent of establishments less than five years old. The establishment size factor includes establishments per employee and the number of establishments with fewer than 20 employees. Establishments per employee (the Glaeser et al. [1992] measure for competition among regional establishments) is selected to represent this factor grouping. The selected measures for industrial specialization and industrial composition (relative wage of occupations in traded industries) do not load on a factor with other measures. These variables are entered individually in the regression analysis.

\subsection{Estimations of Regional Growth Models}

The determinants of regional competitiveness are suggested by regressing the natural logs of the ratios of the 2006 to 2000 values of the competitiveness outcomes (population, employment, and per capita income) on the natural logs of the base year value of the dependent variable, logged current (2006) values of the other two outcome measures, and variables 
representing the groupings of the 1990 initial conditions identified by factor analysis. ${ }^{13}$ The base year for the lagged outcomes is 2000 while 1990 data is used for the independent variables to reduce potential endogeneity bias. The 15-year lag time between the 1990 policy inputs and the 2006 outcomes is consistent with Alcany's (2003) finding that productivity resulting from New Economy information inputs took almost a decade to be realized and did not begin to appear until the late 1990s.

The specific regional adjustment model estimated is provided in equations 12-14:

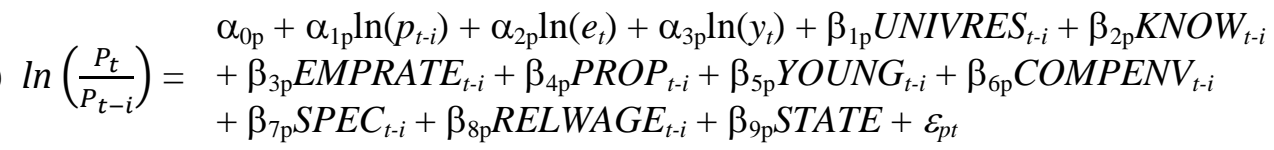

$$
\begin{aligned}
\ln \left(\frac{e_{t}}{e_{t-i}}\right)= & \alpha_{0 \mathrm{e}}+\alpha_{1 \mathrm{e}} \ln \left(p_{t}\right)+\alpha_{2 \mathrm{e}} \ln \left(e_{t-i}\right)+\alpha_{3 \mathrm{e}} \ln \left(y_{t}\right)+\beta_{1 \mathrm{e}} U_{N I V R E S_{t-i}}+\beta_{2 \mathrm{e}} K_{N O W_{t-i}} \\
& +\beta_{3 \mathrm{e}} E M P R A T E_{t-i}+\beta_{4 \mathrm{e}} P R O P_{t-i}+\beta_{5 \mathrm{e}} Y_{\text {OUNG }}+\beta_{6 \mathrm{e}} \text { COMPENV }_{t-i} \\
& +\beta_{7 \mathrm{e}} S P E C_{t-i}+\beta_{8 \mathrm{e}} \text { RELWAGE }_{t-i}+\beta_{9 \mathrm{e}} S T A T E+\varepsilon_{e t}
\end{aligned}
$$

\begin{tabular}{|c|c|}
\hline$p_{t}$ & $=$ population per square mile at time $t$ \\
\hline$p_{t-i}$ & $=$ population per square mile at time $t-i$ \\
\hline$e_{t}$ & $=$ employment per square mile at time $t$ \\
\hline$e_{t-i}$ & $=$ employment per square mile at time $t-i$ \\
\hline$y_{t}$ & $=$ per capita income at time $t$ \\
\hline$y_{t-i}$ & $=$ per capita income at time $t-i$ \\
\hline UNIVRES $_{t-i}$ & $=$ science and engineering graduates at time $t-i$ \\
\hline$K N O W_{t-i}$ & $=$ knowledge workers at time $t-i$ \\
\hline$E M P R A T E_{t-i}$ & $=$ employed/labor force at time $t-i$ \\
\hline$P R O P_{t-i}$ & $=$ proprietors per capita at time $t-i$ \\
\hline COMPENV $V_{t-i}$ & $=$ establishments/employment at time $t-i$ \\
\hline$Y O U N G_{t-i}$ & $=$ percent establishments $<5$ years old at time $t-i$ \\
\hline$S P E C_{t-i}$ & $=$ industrial specialization at time $t-i$ \\
\hline$R E L W A G E_{t-i}$ & $=$ industry relative wage at time $t-i$ \\
\hline STATE & $=$ vector of state dummy variables representing MSA locations \\
\hline & in the estimation of a given outcome at time $t$. \\
\hline
\end{tabular}

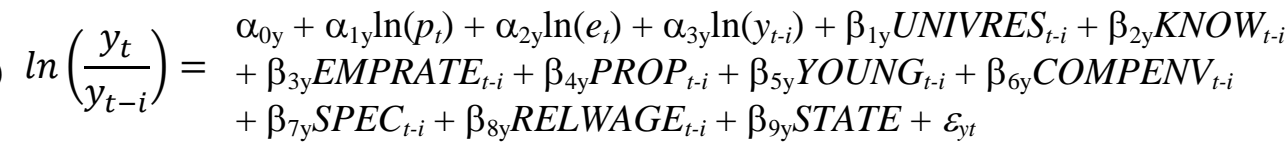

where:

The model in Equations (12) to (14) is estimated using the eight representative variables identified by the factor analysis and the state dummy variables. ${ }^{14}$ The regional adjustment model is estimated using two-stage least squares (2SLS). The first stage estimates the two simultaneous

\footnotetext{
${ }^{13}$ Per capita income is not adjusted for cost of living in the reported estimations. The results of models that relied on adjusted per capita income are not reported due to measurement errors arising from nonreporting cities.

14 Texas was the excluded dummy. Texas had more MSAs (25) than any other state. The Cincinnati-Middletown, OH-IN-KY and Evansville, IN-KY, MSAs were combined with Dover, the lone Delaware MSA, in an "other" dummy. Coefficients on state dummies are not reported as most are insignificant.
} 
outcome levels, and those predicted values are used in the second stage estimation of the change in the growth rate of population, employment, or income. For example, the 2SLS estimation of the population growth rate (Equation 12) includes a first-stage estimation of the 2006 levels of employment and per capita income. The predicted 2006 employment and income values are then used to estimate the change in the population growth rate. ${ }^{15}$

The use of variables selected from the factor analysis decreases the unreliability of regression coefficients caused by multicollinearity. Potential correlation issues are further reduced by using year 2000 values of the lagged, or base year, outcome variables and 1990 values of the remaining explanatory variables. The use of 1990 values for the policy and structure/legacy variables reduces the likelihood of endogeneity bias between explanatory variables (e.g., knowledge workers) and dependent variables (e.g., change in per capita income). ${ }^{16}$ To address heteroskedasticity in the growth variables, White-adjusted standard errors are used to determine the significance of the estimated coefficients.

\section{SUMMARY OF THE FINDINGS}

\subsection{Coefficients on Lagged and Simultaneous Outcome Variables}

Table 3 provides the estimation results of the simultaneous equations for growth rates in metropolitan area population, employment, and per capita income. The coefficients on the logs of the lagged (base year) dependent variables and the logs of the simultaneous outcome variables are interpreted as elasticities. The estimated coefficients on the base year and simultaneous outcome variables have the same signs as found by Carruthers and Mulligan (2008), although the coefficients are not always statistically significant.

The growth rates in population and employment densities are significantly associated with the current values of the metropolitan areas' population density, employment density, and per capita income. Specifically, employment growth rates are higher in areas with relatively high population densities and high per capita incomes. These findings support the hypothesis that job growth is most rapid in areas that provide urbanization economies and high wage employment opportunities. Alternatively, the 2000 to 2006 population growth rates are positively related to area employment levels but negatively associated with the 2006 level of per capita income. This finding of a negative relationship with per capita income may indicate that rapid population growth depresses area income as the labor supply increases or that residential growth is slower in areas with higher costs of living (reflected in per capita income levels). Slower population growth in high per capita income areas may also result from a lower quality of life in the high income metro regions. Roback (1982) suggests that regions with low amenities must compensate with higher wages to attract residents. Finally, growth rates in metropolitan per capita incomes are not significantly associated with current employment density or

\footnotetext{
${ }^{15}$ The regional adjustment model specification is also estimated using a three-stage least squares (3SLS) approach (Kelejian and Prucha, 2004). In 3SLS, specification errors in one equation can bias all outcomes because the third stage enables correlation between the error terms. The results of the 2SLS of 3SLS simultaneous system estimations are compared (1) to ordinary least squares (OLS) regressions (both standard and White-adjusted) of the outcomes on the initial conditions and actual values of the simultaneous level outcomes and (2) to White-adjusted 2SLS estimations in which only 2000 base year outcomes are included on the right hand side of the stage one estimation. The results of all five estimation methods are reasonably similar. The 2 SLS estimations of the simultaneous system produce coefficients and standard errors that are consistent with the alternative specifications. The 3SLS estimations produce coefficients that are larger in absolute value and slightly smaller standard errors.

${ }^{16}$ Regressions estimated using year 2000 values for both the base year outcomes and the initial policy and structure/legacy conditions provide similar results.
} 
TABLE 3. Estimation Results of Regional Adjustment Model

\begin{tabular}{|c|c|c|c|c|c|c|}
\hline Variable & \multicolumn{2}{|c|}{$\ln \left(\mathrm{p}_{06} / \mathrm{p}_{00}\right)$} & \multicolumn{2}{|c|}{$\ln \left(\mathrm{e}_{06} / \mathrm{e}_{00}\right)$} & \multicolumn{2}{|c|}{$\ln \left(\mathrm{y}_{06} / \mathrm{y}_{00}\right)$} \\
\hline Log population/sq. mile, 2006 & -- & & $\begin{array}{c}0.8380 \\
(8.36)\end{array}$ & $* * *$ & $\begin{array}{l}-0.0174 \\
(-0.21)\end{array}$ & \\
\hline Log population/sq. mile, 2000 & $\begin{array}{l}-0.6125 \\
(-8.74)\end{array}$ & $* * *$ & -- & & -- & \\
\hline Log employment/sq. mile, 2006 & $\begin{array}{c}0.6089 \\
(8.99)\end{array}$ & $* * *$ & -- & & $\begin{array}{l}0.0060 \\
(0.07)\end{array}$ & \\
\hline Log employment/sq. mile, 2000 & -- & & $\begin{array}{l}-0.8386 \\
(-8.26)\end{array}$ & $* * *$ & -- & \\
\hline Per capita income, 2006 & $\begin{array}{l}-0.0791 \\
(-1.29)\end{array}$ & & $\begin{array}{l}0.2013 \\
(1.95)\end{array}$ & $*$ & -- & \\
\hline Per capita income, 2000 & -- & & -- & & $\begin{array}{l}-0.1252 \\
(-2.05)\end{array}$ & $* *$ \\
\hline University Research, 1990 & $\begin{array}{l}-0.0036 \\
(-1.06)\end{array}$ & & $\begin{array}{l}0.0110 \\
(1.82)\end{array}$ & $*$ & $\begin{array}{l}-0.0054 \\
(-0.85)\end{array}$ & \\
\hline Knowledge workers, 1990 & $\begin{array}{l}-0.0020 \\
(-0.36)\end{array}$ & & $\begin{array}{l}-0.0104 \\
(-1.13)\end{array}$ & & $\begin{array}{l}0.0225 \\
(2.84)\end{array}$ & $* * *$ \\
\hline Employment/Labor Force, 1990 & $\begin{array}{l}-0.0124 \\
(-1.32)\end{array}$ & & $\begin{array}{l}0.0073 \\
(0.53)\end{array}$ & & $\begin{array}{l}0.0117 \\
(1.62)\end{array}$ & \\
\hline Proprietors per capita, 1990 & $\begin{array}{l}-0.0143 \\
(-2.38)\end{array}$ & $* *$ & $\begin{array}{l}0.0179 \\
(1.58)\end{array}$ & & $\begin{array}{l}0.0035 \\
(0.76)\end{array}$ & \\
\hline Young establishments, 1990 & $\begin{array}{l}0.0200 \\
(2.79)\end{array}$ & $* * *$ & $\begin{array}{l}0.0207 \\
(2.00)\end{array}$ & $* *$ & $\begin{array}{l}0.0034 \\
(0.44)\end{array}$ & \\
\hline Establishments per employee, 1990 & $\begin{array}{l}0.0150 \\
(2.02)\end{array}$ & $* *$ & $\begin{array}{l}-0.0251 \\
(-1.96)\end{array}$ & $*$ & $\begin{array}{l}0.0178 \\
(2.83)\end{array}$ & $* * *$ \\
\hline Industrial specialization, 1990 & $\begin{array}{l}0.0008 \\
(0.23)\end{array}$ & & $\begin{array}{l}-0.0045 \\
(-0.84)\end{array}$ & & $\begin{array}{l}-0.0011 \\
(-0.18)\end{array}$ & \\
\hline Relative industry wage, 1990 & $\begin{array}{l}-0.0061 \\
(-1.56)\end{array}$ & & $\begin{array}{l}0.0022 \\
(0.32)\end{array}$ & & $\begin{array}{l}0.0037 \\
(0.6)\end{array}$ & \\
\hline Constant & $\begin{array}{l}1.3304 \\
(2.17)\end{array}$ & $* *$ & $\begin{array}{l}-2.6523 \\
(-2.63)\end{array}$ & $* * *$ & $\begin{array}{l}1.4260 \\
(2.23)\end{array}$ & $* *$ \\
\hline$R^{2}$ & 0.8433 & & 0.7775 & & 0.4242 & \\
\hline
\end{tabular}

Notes: The number of observations is $151 ; t$-statistics are in parentheses; $* * *$ denotes significance at $p<0.01$; $* *$ denotes significance at $p<0.05 ;$ and $*$ denotes significance at $p<0.10$.

population density, which is inconsistent with the hypothesis of increased labor demand driving wages up and increased labor supply driving wages down.

The 2000-2006 population, employment, and income growth rates are negatively associated with base-year (2000) values. The negative and significant coefficients on the lagged (base year) dependent variables in each equation indicate a hysteresis effect (Cortright, 2001; Nitsch ,2003) consistent with the use of a partial equilibrium model. The base-year coefficients also appear to support the regional convergence hypothesis (Barro, 1991; Glaeser, Scheinkman, and Shleifer, 1995). However, the variances of the logs of population density, employment density, and per capita income all increased between 1990 and 2006, which could indicate 
divergence, as discussed by Drennan (2005). A more nuanced story regarding convergence versus divergence is suggested by comparing the ratios of the top 20 percent of MSAs to the bottom 20 percent for each outcome in 1990, 2000, and 2006. The population ratio rises slightly in each period from 1.3997 in 1990 to 1.4067 in 2006. There is evidence that smaller MSAs grew slower over the period, but there is no evidence that the largest MSAs grew faster. The employment ratio falls from 1.5485 to 1.5239 from 1990 to 2006 while the per capita income ratio falls from 1.0473 in 1990 to 1.0428 in 2000 before rising to 1.0448 in 2006. Each competitiveness outcome appears to have converged or diverged differently; however, the equality ratios were fairly stable over the 2000 to 2006 period, and the short time frame does not facilitate consideration of long-term trends to either convergence or divergence.

Stability tests on modified equations using lagged rather than simultaneous outcomes provide characteristic roots of approximately $0.86,0.86$, and 0.95 for the population, employment, and per capita income growth estimations, respectively. Eigenvectors less than one suggest that the regional adjustment model is stable and reflects a steady-state growth process. The ratios of the characteristic vectors $(0.34: 0.34: 0.32,0.31: 0.31: 0.38,0.43: 0.43: 0.13)$ do not conform to traditional 1:2:1 expectations for a reasonable growth path. Given retirementand amenity-driven migration in the South, it is possible that much employment may be population-driven. The regression coefficients on population and employment also suggest that people follow jobs and jobs follow people. In addition, the time period of this study (2000-2006) coincides with the housing and construction bubble that was a growth stimulus in some metro areas in the South.

\subsection{Competitiveness Inputs and Industry Structure Variables}

The estimated coefficients on the proxy variables for New Economy policy initiatives and local industrial structure indicate that competitiveness outcomes are associated with different inputs and industrial legacies. First, innovative activity (as represented by university graduates in science and engineering) is positively associated with MSA employment growth rates. Places with large numbers of science and engineering graduate students are likely to experience greater job growth as the technology-driven industries that lead the New Economy locate near university research hubs. There is not, however, a significant direct effect on population and income growth rates; positive effects on these outcomes occur primarily as a result of changes in regional employment. These findings are consistent with earlier research indicating that innovation is a critical driver of regional economic growth (Audretsch, 2000; Acs, 2002; Acs et al., 2004).

The growth rate of metropolitan per capita incomes is positively associated with the knowledge worker variable (Florida's "creative class"). As Drennan (2005) points out, human capital is a factor with the potential to raise productivity and wages, and human capital is not subject to diminishing returns. Knowledge workers are often in high-paying occupations and thereby can afford to pay more for goods and services, supporting higher wages across the local economy. These results are consistent with other research indicating an important role for human capital in economic development (Drennan, 2002; Florida, 2002b; Glaeser, 2005). Labor employability (measured by the employment rate) is not significantly associated with any of the 
outcomes. $^{17}$ In sum, it appears that improvements in both innovative activity and human capital are necessarily if more and better jobs are the competitiveness goal.

The role of entrepreneurial activity in enhancing regional competitiveness is less straightforward because three variables reflect different aspects of the MSA entrepreneurial environment and industrial structure or legacy (abundance of proprietors, establishment age, and establishments per employee). Growth rates in MSA population are highest if the MSA is characterized by relatively large numbers of young establishments and establishments per employee (COMPENV). New residents are attracted to regions with newer businesses (with perhaps newer technologies and more appealing work environments) and competitive labor markets (more establishments per worker mean more potential employers). Alternatively, MSA population growth rates are slower if there exists a relatively large number of proprietors. Sole proprietorships may not offer the types of employment opportunities attractive to potential inmigrants, or proprietorship may be a response to a lack of economic opportunities in the slower growing MSAs (Robinson, Dassie, and Christy, 2004).

MSA employment growth rates were positively related to the shares of establishments that are proprietors (PROP) or less than five years old (YOUNG). Davis, Haltiwanger, and Jarmin (2008) and Faberman (2007) also find a strong correlation between community employment growth and young establishments. Young establishments may have higher productivity resulting from the use of newer technologies and production processes. The newer establishments also may have a less developed organizational structure, and thus be more adaptable to changes in the markets. A competitive labor market (establishments per employee) is not, however, conducive to employment growth. Employers are less attracted to MSAs with greater competition among firms for labor and the resulting upward pressure on wages.

MSA growth rates in per capita income are positively related to the number of establishments per employee. That is, a competitive labor market favors the growth of earnings and income in Southern metropolitan areas. No statistically significant association exists between MSA per capita income growth rates and establishment age or ownership type. It is interesting to note that our findings for the association of competitive environment (establishments per employee) with employment and wage growth rates are the reverse of those found by Glaeser et al. (1992) for U.S. metro areas for 1956 to 1987. Glaeser et al. find that establishments per employee are positively associated with employment growth but negatively related to income growth. We suspect that the differences in estimated coefficients result from the different time periods used.

Our findings for the three variables that represent establishment size, age, and ownership type offer interesting insights into the determinants of regional competitiveness. First, the three variables reflect both measures of the entrepreneurial environment (a much promoted "driver" in the New Economy) and legacies of earlier industrial activity. As such, much of an area's entrepreneurial environment is pre-determined by its industrial structure. Second, somewhat surprisingly the three entrepreneur/structure measures are relatively unrelated as indicated by the findings of the factor analysis. MSA's with a large number of proprietors are likely not the same

\footnotetext{
${ }^{17}$ Labor employability was significant in estimations of other specifications of the income model, including the case with weighted factors. The labor employability factor included measures of education, labor force participation, and poverty. These findings may reflect the continued importance of traditional manufacturing and consumer services as sources of jobs in the metropolitan South and are consistent with the explanation provided for a lack of significance in the representative variable model.
} 
MSA's with relatively young establishments which are not necessarily the same MSAs with a relatively large number of smaller establishments. Third, establishment size and age affect metro employment and income growth rates differently. A relatively large number of small establishments are associated with faster income growth but slower employment growth. Alternatively, a relatively large number of young establishments are related to faster employment growth, but no association is evident between establishment age and income growth rates. As such, communities interested in increasing employment should focus on increasing the relative share of proprietors and young establishments. On the other hand, MSA income growth rates are associated primarily with the number of establishments competing for workers (regardless of age or ownership type).

Finally, metropolitan growth rates in employment and income generally are not associated with industrial specialization or composition (relative industry wage) after controlling for other industrial structure and competitiveness input factors. These results may reflect the relative homogeneity of industrial activity in many Southern metropolitan areas. Alternatively, the influences of industrial specialization and composition may be captured by establishment age and size or the region's measure of human capital.

The results of this study reflect relationships for Southern MSAs for the period 2000 to 2006, the time period during which manufacturing employment declined throughout the South and Southern MSAs focused on restructuring to knowledge economy activities. As such, our findings of a significant role for innovation and knowledge workers in employment and income growth are consistent with earlier research for other regions and time periods. We suspect, however, that innovation and knowledge workers may be more strongly related to competitiveness in the South than elsewhere in the nation. Many Southern MSAs have relative low levels of innovative activity and knowledge workers, and communities successful in increasing these competitiveness inputs (e.g. Austin, TX and Raleigh-Durham, NC) are wellpositioned to attract firms and households.

\section{CONCLUSION}

Innovation inputs, knowledge workers, labor employability, and entrepreneurial environment are shown to be related to growth rates of population, employment, and per capita income. However, the coefficients have different values and levels of significance in estimations of the three outcomes. For example, innovation has a positive association with employment growth, while knowledge workers have a positive association with per capita income growth. The results suggest that different policy approaches are appropriate in achieving different policy goals.

Our findings for Southern metropolitan areas are consistent with earlier studies suggesting that regional competitiveness is a complicated process that requires a multifaceted approach (e.g., Camagni, 2002; Kitson, Martin, and Tyler, 2004; Malecki, 2004; and Eberts, Erickcek, and Kleinhenz, 2006). That is, if regional competitiveness is defined as enhancing a region's attractiveness to residents and businesses, then programs to promote competitiveness must address growth in jobs, residents, and well being. No single input of the process (innovation, human capital, or entrepreneurship) is associated with all of the competitiveness outcomes (population, jobs, and income). Thus, regional competitiveness policies for the New Economy must include efforts to increase innovative activity, enhance the quality of human capital, and improve the entrepreneurial environment. 
Although potentially powerful in their own right, such policies can also complement the industrial recruitment strategies traditionally favored in the South. Henderson and Weiler (2010) note that the cultivation of innovation and entrepreneurship are long-run initiatives that are most effective in densely populated metropolitan areas. The smaller metro areas in the South likely will need to continue their industrial recruitment programs as they work to improve the local environment for innovation and entrepreneurship. Manufacturing activities remaining in the U.S. have become more technologically sophisticated and require skilled labor. Thus, New Economy development policies aimed at building human capital and other innovation-based infrastructure may benefit industrial recruitment efforts. At the same time, Southern states' typically favorable tax and labor laws may provide added incentives for both firms and households.

Our findings for Southern metro areas are similar to earlier research indicating that legacy of place is strongly associated with area changes in employment and population (Eberts, Erickcek, and Kleinhenz, 2006). A region's industrial structure (particularly establishment age and size) influences regional competitiveness, and policy makers will encounter hysteresis effects of industrial legacy. These conclusions are expected to hold across other regions of the U.S. However, regions are expected to be affected by their legacies differently. For example, the Northeast might experience significant positive effects on population and employment from specialization in New Economy industries such as computer components while the Midwest might experience significant negative effects on both employment and income as a result of specialization in more traditional manufacturing activities. Policy makers can also influence industrial structure by encouraging formation and/or recruitment of new firms. Of course, as Drennan (2002) notes, no single entity governs the various cities of most MSAs. The cooperation needed to ensure regional competitiveness is thus a source of future research.

In summary, researchers and policy makers use a variety of concepts and measures when they refer to the competitiveness of a region or relative competitiveness rankings among regions. This study indicates that discussions of regional competitiveness need greater focus on the outcomes of economic growth and development and less attention on competitiveness as a goal. Indeed, polices to enhance regional competitiveness would be more easily identified and implemented if the term competitiveness were dropped and discussion targeted the region's specific development goals (more people, more jobs, higher incomes). For example, an area desiring higher per capita incomes should focus on measures to improve local human capital, while a region wishing more rapid job growth should concentrate its efforts on enhancing entrepreneurship and innovative activity.

Finally, policymakers need to appreciate the roles of industrial structure and composition in the regional economy. The impacts of changes in innovation, human capital, and entrepreneurship on competitiveness (however defined by the region) will be significantly influenced by the industrial legacy of the region. An appreciation for the area's economic history may lead to greater efficiency in economy policy selection and implementation.

\section{REFERENCES}

Acs, Zoltan J. (2002) Innovation and the Growth of Cities. Edward Elgar: Norhampton, MA.

Acs, Zoltan J., David B. Audretsch, Pontus Braunerhjelm, and Bo Carlsson. (2004) "The Missing Link: The Knowledge Filter and Entrepreneurship in Economic Growth," Center for 
Economic Policy Research Working Paper No. 4358, last accessed March 2011 at http://ideas.repec.org/p/cpr/ceprdp/4783.html.

Alcany, Roger. (2003) The New Economy. Farrar, Straus and Giroux: New York.

American Chamber of Commerce Researchers Association. (1990, 2000, 2006) ACCRA Cost of Living Index, First Quarter 1990. C2ER: The County for Community and Economic Research: Arlington, VA.

Atkinson, Robert D. (1990) "Understanding Business Climate Studies: Their Use and Validity," Economic Development Review, Winter, 46-49.

Atkinson, Robert D. and Daniel K. Correa. (2007) The 2007 State New Economy Index: Benchmarking Economic Transformation in the States. Ewing Marion Kauffman Foundation: Kansas City, MO. Last accessed March 2011 at http://www.itif.org/files/2007 State New Economy Index.pdf.

Atkinson, Robert D. and Randolph H. Court. (1998) “The New Economy Index: Understanding America's Economic Transformation," Progressive Policy Institute: Washington, DC. last accessed July 2008 at http://www.neweconomyindex.org/states.

Atkinson, Robert D. and Paul. D. Gottlieb. (2001) The Metropolitan New Economy Index: Benchmarking Economic Transformation in the Nation's Metropolitan Areas. Progressive Policy Institute: Washington, DC. Last accessed March 2011 at http://www.research.fsu.edu/techtransfer/documents/mnei.pdf

Audretsch, David B. (2000) "Knowledge, Globalization, and Regions: An Economists' Perspective," in John H. Dunning (ed.), Regions, Globalization, and the KnowledgeBased Economy. Oxford University Press: Oxford, U.K., pp. 63-82.

Austrian, Ziona, Iryna Lendel, and Afia Yamoah. (2007) An Update of the Regional Growth Model for Large and Mid-Size U.S. Metropolitan Areas: Northeast Ohio Dashboard Indicators. Center for Economic Development, Cleveland State University. Last accessed March 2011 at http://urban.csuohio.edu/publications/center/center for economic development/das hboard report final 0807.pdf

Bao, Shuming, Mark S. Henry, and David L. Barkley. (2004) "Identifying Urban-Rural Linkages: Tests for Spatial Effects in the Carlino-Mills Model," in Luc Anselin, Raymond J.G.M. Florax, and Sergio Rey (eds.), Advances in Spatial Econometrics: Methodology, Tools, and Applications. Springer-Verlag: New York, pp. 321-333.

Barkley, David L. (2001) "Employment Generation Strategies for Small Towns: An Overview of the Alternatives," Regional Economic Development Research Laboratory Report 092001-02, Clemson University. Last accessed March 2011 at http://cherokee.agecon.clemson.edu/redrl rpt2.pdf.

Barkley, David L., and Mark S. Henry. (2001) “Advantages and Disadvantages of Targeting Industry Clusters," Regional Economic Development Research Laboratory Report 092001-01. last accessed November 2010 at http://cherokee.agecon.clemson.edu/redrl.htm.

Barney, Jay B. (1991) "Firm Resources and Sustained Competitive Advantage," Journal of Management, 17, 99-120. 
Barro, Robert J. (1991) "Economic Growth in a Cross-Section of Countries," Quarterly Journal of Economics, 106, 407-444.

Boarnet, Marlon G., Saksith Chalermpong, and Elizabeth Geho. (2005) "Specification Issues in Models of Population and Employment Growth," Papers in Regional Science, 84, 21-46.

Bristow, Gillian. (2005) "Everyone's a 'Winner': Problematising the Discourse of Regional Competitiveness," Journal of Economic Geography, 5, 285-304.

Budd, Leslie, and Amer K. Hirmis. (2004) "Conceptual Framework for Regional Competitiveness," Regional Studies, 38, 1015-1028.

Camagni, Roberto. (2002) "On the Concept of Territorial Competitiveness: Sound or Misleading?," Urban Studies, 39, 2395-2411.

Camp, S. Michael. (2005) "The Innovation-Entrepreneurship NEXUS: A National Assessment of Entrepreneurship and Regional Economic Growth and Development," Small Business Research Summary No. 256. Small Business Administration: Washington, DC.

Carlino, Gerald A. and Edwin S. Mills. (1987) “The Determinants of County Growth,” Journal of Regional Science, 27, 39-54.

Carruthers, John I. and Gordon F. Mulligan. (2008) "A Location al Analysis of Growth and Change in American Metropolitan Areas," Papers in Regional Science, 87, 155-171.

Clark, David E. and Christopher A. Murphy. (1996) "Countywide Employment and Population Growth: An Analysis of the 1980s," Journal of Regional Science, 36, 235-256.

Clinton, Jim and Carol Conway with Scott Doron, Linda Hoke, and Keecia James. (2002) The Mercedes and the Magnolia: Preparing the Southern Workforce for the New Economy, The 2002 Report on the Future of the South. Southern Growth Policies Board: Research Triangle Park, NC. Last accessed March 2011 at http://www.southerngrowth.com/pubs/pubs pdfs/rfs2002.pdf.

Combs, Susan. (2009) Texas Comptroller of Public Accounts Window on State Government Web site. Last accessed November 2010 at http://www.window.state.tx.us.

Corporation for Enterprise Development. (2003, 2007) "Development Report Card for the States: Measures," Corporation for Enterprise Development: Washington, DC. Last accessed March 2011 at http://cfed.org/knowledge center/research/DRC/.

Cortright, Joseph. (2001) "New Growth Theory, Technology and Learning: A Practitioner's Guide," U.S. Economic Development Administration, Reviews of Economic Development Literature and Practice, No. 4. Last accessed March 2011 at http://www.eda.gov/ImageCache/EDAPublic/documents/pdfdocs/1g3lr $5 f 75$ fcortright 2epdf/v1/1g3/r $5 f 7$ ffcortright.pdf.

Davis, Steven J., John Haltiwanger, and Ron Jarmin. (2008) Turmoil and Growth: Young Businesses, Economic Churning, and Productivity Gains. Ewing Marion Kauffman Foundation: Kansas City, MO. Last accessed March 2011 at http://sites.kauffman.org/pdf/TurmoilandGrowth060208.pdf. 
Deller, Steven C., Tsung-Hsiu Tsai, David W. Marcouiller, and Donald B.K. English. (2001) "The Role of Amenities and Quality of Life in Rural Economic Growth," American Journal of Agricultural Economics, 83, 352-365.

DeVol, Ross, Armen Bedroussian, and Soojung Kim. (2007) Best Performing Cities 2007: Where America's Jobs Are Created and Sustained. Milken Institute: Santa Monica, CA. Last accessed March 2011 at http://www.milkeninstitute.org/pdf/best prfrmg cities.pdf.

Dissart, Jean-Christophe. (2003) "Regional Economic Diversity and Regional Economic Stability: Research Results and Agenda," International Regional Science Review, 26, 423-446.

Doron, Scott, Linda Hoke, Sandra Johnson, Charity Pennock, and Jim Clinton. (2006) Innovation with a Southern Accent: The 2006 Report on the Future of the South. Southern Growth Policies Board: Research Triangle Park, NC. Last accessed March 2011 at http://www.southerngrowth.com/pubs/pubs pdfs/rfs2006.pdf.

Drennan, Matthew J. (1999) "National Structural Change and Metropolitan Specialization in the United States," Papers in Regional Science, 78, 297-318.

. (2002) The Information Economy and American Cities. The Johns Hopkins University Press: Baltimore, MD.

- (2005) "Possible Sources of Wage Divergence among Metropolitan Areas of the United States," Urban Studies, 42, 1609-1620.

Eberts, Randall W., George A. Erickcek, and John Kleinhenz. (2006) "Dashboard Indicators for the Northeast Ohio Economy: Prepared for the Fund for Our Economic Future," Federal Reserve Bank of Cleveland Working Paper No. 06-05. Last accessed March 2011 via http://ssrn.com/abstract=1022345.

European Commission. (1999) Sixth Periodic Report on the Social and Economic Situation of Regions in the EU. European Commission: Brussels.

Faberman, R. Jason. (2007) "The Relationship between the Establishment Age Distribution and Urban Growth," Federal Reserve Bank of Philadelphia Working Paper No. 07-18. Last accessed March 2011 at https://www.phil.frb.org/research-and-data/publications/workingpapers/2007/wp07-18.pdf.

Fairlie, Robert W. and Alicia M. Robb. (2008) Race and Entrepreneurial Success: Black-, Asian-, and White-Owned Businesses in the United States. MIT Press: Cambridge, MA.

Fisher, Peter. (2005) Grading Places: What Do Business Climate Rankings Really Tell Us? Economic Policy Institute: Washington, DC.

Florida, Richard. (2002a) "Bohemia and Economic Geography," Journal of Economic Geography, 2, 55-71.

. (2002b) The Rise of the Creative Class... and How It's Transforming Work, Leisure, Community and Everyday Life. Basic Books: New York.

Gabe, Todd M. (2006) "Growth of Creative Occupations in U.S. Metropolitan Areas: A ShiftShare Analysis, Growth and Change, 37, 396-415. 
Gardiner, Ben. (2003) "Regional Competitiveness Indicators for Europe - Audit, Database Construction and Analysis," unpublished paper presented at the Regional Studies Association International Conference, Pisa, Italy, April 15. Last accessed March 2011 at http://94.76.226.154/Libraries/Downloadable Files/Regional Competitiveness Indicato rs for Europe PDF.sflb.ashx.

Gardiner, Ben, Ron Martin, and Peter Tyler. (2004) "Competitiveness, Productivity and Economic Growth across the European Regions," Regional Studies, 38, 1045-1067.

Glaeser, Edward L. (2005) “Book Review: Edward L. Glaeser, Review of Richard Florida's The Rise of the Creative Class," Regional Science and Urban Economics, 35, 593-596.

Glaeser, Edward L., José A. Scheinkman, and Andrei Shleifer. (1995) "Economic Growth in a Cross-Section of Cities," Journal of Monetary Economics, 36, 117-143.

Glaeser, Edward L., Hedi D. Kallal, Jose A. Scheinkman, and Andrei Shleifer. (1992) "Growth in Cities," Journal of Political Economy, 100, 1126-1152.

Glavac, Sonya M., Alexander C. Vias, and Gordon F. Mulligan. (1998) "Population and Employment Interactions in the Growth of United States Micropolitan Centers," Urban Geography, 19, 632-656.

Graham, Bart L. (2011) 2010 Tax Guide for Georgia Citizens. Department of Revenue, State of Georgia. Last accessed March 2011 at https://etax.dor.ga.gov/taxguide/TSD Tax Guide for Georgia Citizens 2010.pdf

Greene, Francis J., Paul Tracey, and Marc Cowling. (2007) "Recasting the City into CityRegions: Place Promotion, Competitiveness Benchmarking and the Quest for Urban Supremacy," Growth and Change, 38, 1-22.

Greene, William H. (2003) Econometric Analysis, $5^{\text {th }}$ Ed. Pearson Education: Patparganj, India.

Harvard Business School, Institute for Strategy and Competitiveness. (2008) Cluster Mapping Project. Last accessed March 2011 at https://secure.hbs.edu/isc/login/login.do?http://data.isc.hbs.edu/isc/index.jsp.

Harvey, David. (1989) "From Managerialism to Entrepreneurialism: The Transformation of Urban Governance in Late Capitalism," Geografiska Annaler, 71 B, 3-17.

Hatcher, Larry. (1994) A Step-by-Step Approach to Using SAS® for Factor Analysis and Structural Equation Modeling. SAS Institute, Inc.: Cary, NC.

Haughton, Jonathan and Corina Murg. (2002) Metro Area and State Competitiveness Report 2002. The Beacon Hill Institute for Public Policy Research, Suffolk University. Last accessed March 2011 at http://www.beaconhill.org/BHIStudies/Compete2002Data/Compete2002Web.pdf.

Henderson, Jason and Stephan Weiler. (2010) "Entrepreneurs and Job Growth: Probing the Boundaries of Time and Space," Economic Development Quarterly, 24, 23-32.

Henry, Mark S., Bertrand Schmitt, Knud Kristenson, David L. Barkley, and Shuming Bao. (1999) "Extending Carlino-Mills Models to Examine Urban Size and Growth Impacts on Proximate Rural Areas," Growth and Change, 30, 526-548. 
Henry, Mark S., Bertrand Schmitt, and Virginie Piguet. (2001) "Spatial Econometric Models for Simultaneous Equations Systems: An Application to Rural Community Growth in France," International Regional Science Review, 24, 171-193.

Huggins, Robert. (2003) "Creating a UK Competitiveness Index: Regional and Local Benchmarking," Regional Studies, 37, 89-96.

Intriligator, Michael D. (1978) Econometric Models, Techniques, and Applications. PrenticeHall: Englewood Cliffs, NJ.

Kelejian, Harry H., and Ingmar R. Prucha. (2004) "Estimation of Simultaneous Systems of Spatially Interrelated Cross Sectional Equations," Journal of Econometrics, 118, 27 - 50

Kim, Jae-On, and Charles W. Mueller. (1978) "Factor Analysis: Statistical Methods and Practical Issues." Sage University Quantitative Applications in the Social Sciences Series Paper 07-014.

Kitson, Michael, Ron Martin, and Peter Tyler. (2004) "Regional Competitiveness: An Elusive yet Key Concept?” Regional Studies, 38, 991-999.

Krugman, Paul. (1990) The Age of Diminished Expectations. MIT Press: Cambridge, MA. . (1991) Geography and Trade. Leuven University Press: Leuven, Belgium. . (1996) "Making Sense of the Competitiveness Debate," Oxford Review of Economic Policy, 12, 17-25.

Ma, Hao. (2000) "Competitive Advantage and Firm Performance," Competitiveness Review, 10, 15-32.

Malecki, Edward J. (2004) "Jockeying for Position: What It Means and Why It Matters to Regional Development Policy When Places Compete,” Regional Studies, 38, 1101-1120.

Markusen, Ann R. (1996) "Sticky Places in Slippery Space: A Typology of Industrial Districts," Economic Geography, 72, 293-313.

Mueser, Peter R., and Philip E. Graves. (1995) "Examining the Role of Economic Opportunity and Amenities in Explaining Population Redistribution," Journal of Urban Economics, 37, 176-200

National Competitiveness Council. (2007) "Review of International Assessments of Ireland's Competitiveness," Forfás: Dublin, Ireland. Last accessed March 2011 at http://www.forfas.ie/media/ncc071220 international competitiveness review1.pdf.

Nitsch, Volker. (2003) "Does History Matter for Urban Primacy? The Case of Vienna," Regional Science and Urban Economics 33, 401-418.

Norton, R.D. (2000) "The Geography of the New Economy," in Scott Loveridge (ed.), The Web Book of Regional Science. Regional Research Institute: Morgantown, WV. Last accessed March 2011 at http://www.rri.wvu.edu/WebBook/Norton/sectiona.htm.

Porter, Michael E. (1990) The Competitive Advantage of Nations. MacMillan: London. . (2002) "Regional Foundations of Competitiveness and Implications for Government Policy," paper presented at the Department of Trade and Industry Workshop on Regional Competitiveness, April. 
. (2003) “The Economic Performance of Regions,” Regional Studies, 37, 549-578.

Roback, Jennifer. (1982) "Wages, Rents, and the Quality of Life," Journal of Political Economy, $90,1257-1278$.

Robinson, Kenneth L., Wylin Dassie, and Ralph D. Christy. (2004) "Entrepreneurship and Small Business Development as a Rural Development Strategy," Southern Rural Sociology, 20(2), 1-27.

Rork, Jonathan C. (2005) "Getting What You Pay For: The Case of Southern Economic Development," Journal of Regional Analysis and Policy, 35, 37-53.

Schumpeter, Joseph A. (1942) Capitalism, Socialism, and Democracy. Harper Books: New York.

Scott, Bruce R. and George C. Lodge, eds. (1985) U.S. Competitiveness and the World Economy. Harvard Business School Press: Boston.

Shaffer, Sherrill. (2002) "Firm Size and Economic Growth," Economic Letters, 76, 195-203.

StataCorp. (2005) Stata Statistical Software: Release 9. StataCorp LP: College Station, TX.

Steinnes, Donald N., and Walter D. Fisher. (1974) "An Econometric Model of Interurban Location," Journal of Regional Science, 14, 65-80.

Steinle, Wolfgang J. (1992) "Regional Competitiveness and the Single Market," Regional Studies, 26, 307-318.

Storper, Michael. (1997) The Regional World: Territorial Development in a Global Economy. Guildford Press: New York.

Taylor, Ray, Sandra Johnson, Linda Hoke, Scott Doron, Charity Pennock, and Jim Clinton. (2007) Enterprise South.biz: The 2007 Report on the Future of the South. Southern Growth Policies Board: Research Triangle Park, NC. Last accessed March 2011 at http://www.southerngrowth.com/pubs/pubs_pdfs/rfs2007_execsum.pdf.

Tuerck, David G., Jonathan Haughton, Frank Conte, and Christopher Doyon. (2007) State Competitiveness Report 2007. The Beacon Hill Institute for Public Policy Research, Suffolk University. Last accessed March 2011 at http://www.beaconhill.org/Compete07/Compete2007State.pdf.

Tuerck, David G., Jonathan Haughton, Frank Conte, and Ashley Mowatt. (2008) Metro Area Competitiveness Report 2007. The Beacon Hill Institute for Public Policy Research, Suffolk University. Last accessed March 2011 at http://www.beaconhill.org/Compete07/Compete2007MetroBHI.pdf.

Wheeler, Christopher H. (2006) "Human Capital Growth in a Cross Section of U.S. Metropolitan Areas," Federal Reserve Bank of St. Louis Review, March/April, 113-132. 


\section{APPENDIX TABLE A1. Orthogonally Rotated Factor Loadings from Standardized Variables}

\begin{tabular}{|c|c|c|c|c|c|c|c|}
\hline \multirow[b]{2}{*}{ Variable } & \multicolumn{7}{|c|}{ Factor } \\
\hline & $\begin{array}{l}\text { Innovation } \\
\text { Inputs }\end{array}$ & $\begin{array}{l}\text { Knowledge } \\
\text { Workers }\end{array}$ & $\begin{array}{c}\text { Labor } \\
\text { Employability } \\
\end{array}$ & $\begin{array}{c}\text { Entrepreneurial } \\
\text { Environment }\end{array}$ & $\begin{array}{c}\text { Young } \\
\text { Establishments } \\
\end{array}$ & $\begin{array}{c}\text { Small } \\
\text { Establishments } \\
\end{array}$ & $\begin{array}{c}7 / 8 \\
\text { Independent } \\
\text { Structure \& } \\
\text { Legacy } \\
\text { Variables } \\
\end{array}$ \\
\hline Graduate students in science and engineering per 10,000 residents & 0.9761 & 0.0768 & -0.0292 & 0.0019 & 0.0042 & -0.0065 & 0.0404 \\
\hline Science and engineering PhD's per 10,000 residents & 0.9685 & 0.0792 & -0.0189 & 0.0022 & 0.0086 & -0.0254 & 0.0546 \\
\hline Academic R\&D spending per capita & 0.9446 & 0.0914 & -0.005 & -0.0084 & 0.0213 & 0.0123 & 0.0987 \\
\hline College and graduate school enrollment per 10,000 residents & 0.9285 & 0.1255 & -0.0719 & -0.0645 & -0.0405 & 0.0085 & 0.1111 \\
\hline Percent of population ages $25+$ with an advanced degree & 0.7906 & 0.4765 & 0.117 & 0.0083 & 0.0809 & 0.0671 & 0.1231 \\
\hline Percent of employment in computer, science, and engineering occupations & 0.6251 & 0.6561 & 0.0296 & -0.0792 & -0.1278 & 0.0619 & 0.1514 \\
\hline $\begin{array}{l}\text { Percent of employment in management, business/operations, finance, } \\
\text { computers, math, architecture, engineering, sciences, law, healthcare, } \\
\text { arts, design, entertainment, media, education, and high-end sales } \\
\text { occupations }\end{array}$ & 0.3137 & 0.8923 & 0.0139 & 0.0354 & 0.2115 & -0.0595 & 0.0557 \\
\hline $\begin{array}{l}\text { Percent of employment in professional, scientific, and technical services } \\
\text { industries }\end{array}$ & 0.062 & 0.8770 & 0.0558 & 0.2215 & 0.2030 & -0.0587 & 0.1302 \\
\hline Percent of employment in manufacturing sectors & -0.0684 & -0.7031 & 0.2457 & -0.0177 & -0.1723 & 0.5164 & 0.1439 \\
\hline Percent of population ages $25+$ with a bachelor's degree & 0.3797 & 0.7503 & 0.2076 & 0.1588 & 0.2529 & 0.2730 & 0.0860 \\
\hline Percent of population ages $25+$ with less than a high school diploma & -0.1289 & -0.6769 & -0.5636 & 0.1210 & -0.0437 & 0.2365 & 0.1350 \\
\hline Percent of population of working age (25-64) & -0.319 & 0.1568 & 0.5361 & 0.1105 & -0.0713 & 0.5305 & 0.2876 \\
\hline Labor force participation rate & 0.1491 & 0.1274 & 0.5841 & -0.1184 & 0.1110 & 0.4986 & 0.3455 \\
\hline Employment rate (employed/labor force) & 0.1334 & -0.0080 & 0.8536 & 0.0306 & 0.2140 & 0.1823 & 0.1736 \\
\hline Percent of population who speak English well & 0.0545 & 0.0201 & 0.7072 & -0.1850 & -0.3915 & 0.0256 & 0.3083 \\
\hline Out-of-poverty rate & -0.2064 & 0.0655 & 0.9329 & 0.0946 & 0.0774 & 0.0769 & 0.0619 \\
\hline Number of proprietors per capita & 0.0271 & 0.2715 & 0.1896 & 0.7301 & -0.0185 & -0.034 & 0.3551 \\
\hline Proprietors' income as a share of total earnings & -0.1348 & 0.1582 & -0.2585 & 0.7250 & -0.0290 & -0.1599 & 0.3380 \\
\hline Percent of population that is a racial minority & -0.0082 & 0.3048 & -0.3034 & -0.4852 & 0.0278 & 0.2494 & 0.5166 \\
\hline Percent of establishments less than five years old & 0.0062 & 0.2711 & 0.1447 & -0.0099 & 0.8438 & -0.1977 & 0.1543 \\
\hline Business churning & -0.0289 & 0.3349 & 0.0229 & -0.0539 & 0.7857 & -0.2092 & 0.2224 \\
\hline Establishments per employee & -0.0601 & 0.1651 & -0.1223 & 0.0930 & 0.2527 & -0.8720 & 0.1213 \\
\hline Percent of establishments with fewer than 20 employees & -0.0164 & 0.0293 & -0.0437 & 0.1004 & 0.1141 & -0.8453 & 0.2593 \\
\hline Employment specialization index & 0.2744 & -0.4631 & 0.0598 & 0.0257 & 0.2765 & -0.1504 & 0.6069 \\
\hline Relative wage of occupations in traded industries & 0.0599 & 0.3603 & 0.2736 & 0.0984 & 0.0512 & 0.4224 & 0.6010 \\
\hline
\end{tabular}

(C) Southern Regional Science Association 2011.

ISSN 1553-0892

SRSA, 1601 University Avenue, PO Box 6025, Morgantown, West Virginia 26506-6025, USA. 